\title{
NSM CFRP Strips for Shear Strengthening of RC Beams: Tests and Me- chanical Model
}

\author{
J. A. O. Barros ${ }^{*}, 1$, Vincenzo Bianco ${ }^{2}$ and Giorgio Monti ${ }^{3}$
}

\author{
${ }^{1}$ Associate Prof., Dept. of Civil Eng., Univ. of Minho, Azurém, 4810058 Guimarães, Portugal; ${ }^{2}$ PhD Student, Dept. of \\ Structural Eng. and Geotechnics, Univ. of Roma "La Sapienza", 00197 Roma, Italy; ${ }^{3}$ Full Prof., Dept. of Structural \\ Eng. and Geotechnics, Univ. of Roma "La Sapienza”, 00197 Roma, Italy
}

\begin{abstract}
The application of Carbon Fiber Reinforced Polymer (CFRP) strips according to the Near Surface Mounted (NSM) technique has proven to be a promising shear strengthening strategy for RC beams, in terms of effectiveness and executability. Nevertheless, several aspects concerning the underlying resisting mechanisms and their mechanical interpretation still need to be clarified and organized in a comprehensive model. By a critical overview of the relevant research findings available to date in the analytical modeling domain, it emerges that most of the efforts carried out are mainly devoted to quantify parameters related to the NSM debonding failure mechanism, on the basis of test set-ups whose geometry often greatly differs from the actual conditions met in a common T-cross section beam. To give some contribution for the discussion of these subjects, an experimental program was carried out, on T-beams of quasi-real scale and with a given ratio of existing steel stirrups. The main results are presented and analyzed in the present work.

In the second part of this work, a new analytical predictive model is proposed. It assumes as possible failure mechanisms: debonding, tensile rupture of the strip and the concrete tensile fracture and allows the interaction between strips to be accounted for. The comparison between the results determined by the application of the proposed model and those obtained from experimental research reveals the high predictive accuracy of this model.
\end{abstract}

Keywords: Near Surface Mounted, CFRP, Shear Strengthening, Debonding, Concrete, Critical Diagonal Crack.

\section{INTRODUCTION}

The possibilities of a technique, designated as Near Surface Mounted (NSM), for the shear strengthening of reinforced concrete (RC) beams was started being explored at the beginning of this century [1]. This technique consists on fixing, with epoxy adhesive, fiber reinforced polymer (FRP) bars into grooves opened in the concrete cover of the beam lateral faces. In this exploratory work round bars were used but, recently, the higher effectiveness of square bars was proved [2]. To assess the effectiveness of the NSM technique for the shear strengthening of RC beams, using carbon FRP (CFRP) strips of rectangular cross section, Barros and Dias [3] carried out an experimental program to analyze the influences of the strips' inclination, beam depth and longitudinal tensile steel reinforcement ratio on the effectiveness of the externally bonded reinforcement (EBR) and NSM strengthening techniques. Amongst the CFRP strengthening techniques, the NSM with strips at $45^{\circ}$ resulted to be the most effective, not only in terms of shear resistance increment but also in terms of deformation capacity at failure of the beams. The NSM was also faster and easier to apply than the EBR technique. To simulate the contribution of the NSM strips for the shear strengthening of tested beams, those authors applied the debonding-based formulation proposed by Nanni

*Address correspondence to this author at the Dept. of Civil Eng., Univ. of Minho, Azurém, 4810058 Guimarães, Portugal;

E-mail: barros@civil.uminho.pt et al. [4], with some adjustments in order to take into account the specificities related to the use of strips instead of round bars [5]. The predictive performance of this model can be found elsewhere [5]. Despite the improvements introduced, the existing Debonding-based analytical predictive Model (DM) systematically provided an overestimation, the higher the smaller the spacing, of the experimentally recorded shear strengthening contribution by NSM CFRP strips. Such overestimation, as further confirmed by experimental evidence, can be ascribed to the erroneous assumption that the expected failure mechanism is debonding, regardless of the influence of concrete tensile strength, interaction between consecutive strips, and existing stirrups/strips interaction.

The analysis of the failure modes of the beams of the experimental programs carried out by Barros and Dias [3] and Dias and Barros [6], has made clear that it is not possible to extend the debonding-based analytical predictive models to NSM. In fact, in the beams with smaller strip spacing the lateral concrete cover of the web separated from the beam concrete core, indicating that the concrete tensile strength plays a paramount role, by limiting the contribution of these systems to the shear strengthening of RC beams. To give some contribution for the discussion of these subjects, an experimental program was carried out, with T-beams of quasi-real scale and with a given ratio of existing steel stirrups. The main results are presented and analyzed in the present work. At the same time a new model is proposed in this work, able of capturing the essential phenomena involved in 

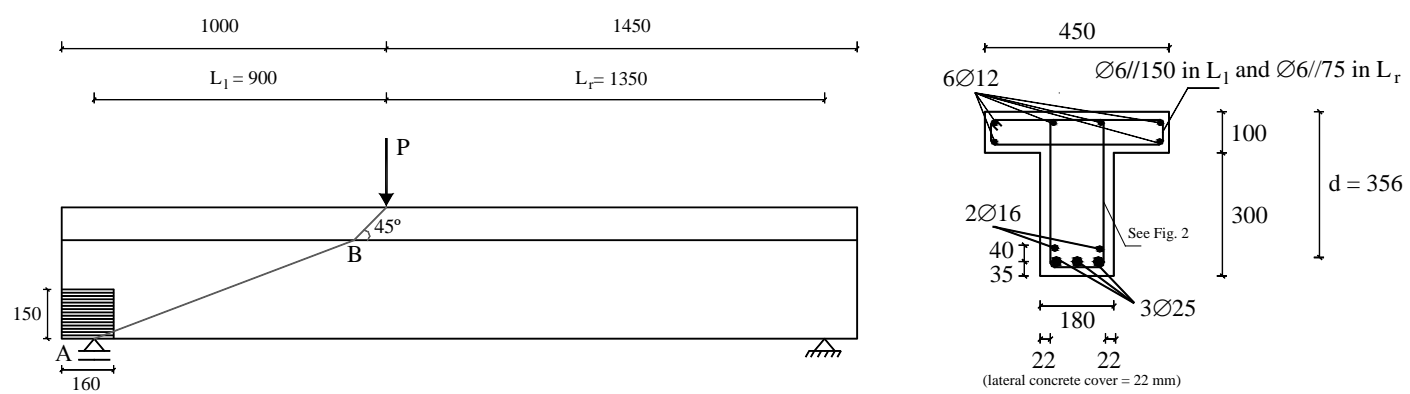

Fig. (1). Beam prototype: geometry, steel reinforcements, load and support conditions.

this strengthening technique, namely: debonding; interaction between strips; concrete tensile fracture; tensile rupture of the strips. This model is described in this work and its performance is assessed taking the obtained experimental results.

\section{EXPERIMENTAL PROGRAM}

\section{Test Series, Strengthening Technique, Test Setup and Material Properties}

The T-cross section of the twelve RC beams composing the experimental program is represented in Fig. (1). The re-

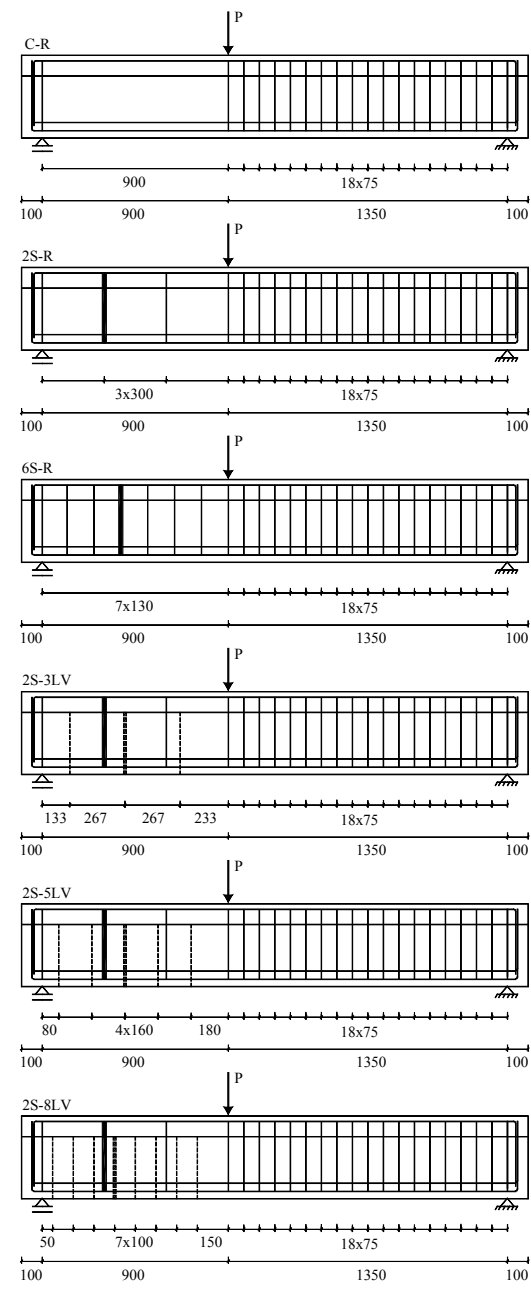

inforcement was designed to activate shear failure for all tested beams. To have shear failure in only one half-span, a non-symmetric three point load configuration with two different shear spans was chosen and high transverse reinforcement (steel stirrups of $6 \mathrm{~mm}$ diameter spaced at $75 \mathrm{~mm}$ - $\phi 6 @ 75 \mathrm{~mm}$ ) was placed in the larger beam span $L_{r}$, as (Fig. 2) shows. The monitored shorter beam span $\left(L_{l}\right)$ where shear failure should occur, had a "shear span-to-depth" ratio of $L_{l} / d=2.5$, where $d$ is the beam effective depth (Fig. 1).

The experimental program (see Table 1) was composed of one beam with no shear reinforcement (C_R beam), one

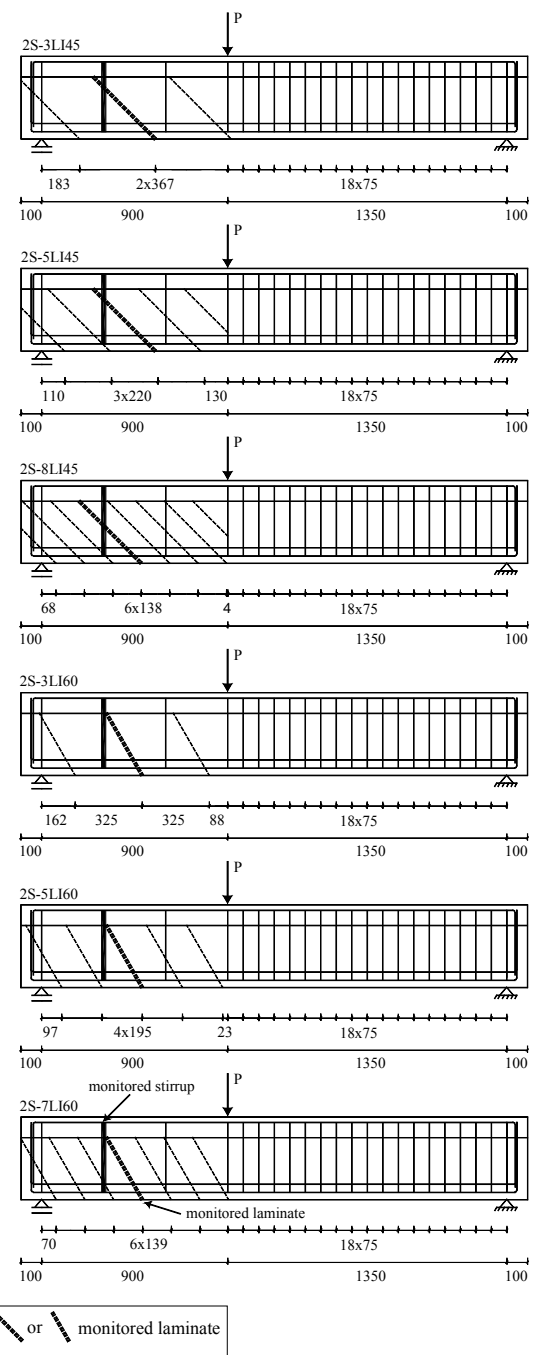

Fig. (2). Tested beams: position of the steel stirrups (thick line) and strips (dashed line). 
Table 1. Shear Reinforcement and Strengthening Systems in the Tested Beams

\begin{tabular}{|c|c|c|c|c|c|}
\hline Beam label & $\begin{array}{l}\text { Age at beam test } \\
\text { [days] }\end{array}$ & \multicolumn{4}{|c|}{ Shear reinforcement/strengthening in the smaller shear span $\left(L_{l}\right)$} \\
\hline C_R & 65 & - & - & - & - \\
\hline $2 \mathrm{~S} \_\mathrm{R}$ & 61 & Steel stirrups & $2 \phi 6$ with two legs $(0.10)$ & 300 & 90 \\
\hline 6S_R & 62 & Steel stirrups & $6 \phi 6$ with two legs $(0.24)$ & 130 & 90 \\
\hline $2 \mathrm{~S} \_3 \mathrm{LV}$ & 72 & CFRP strips & $2 \times 3$ strips with $1.4 \times 10 \mathrm{~mm}^{2}(0.06)$ & 267 & 90 \\
\hline \multirow{2}{*}{$2 \mathrm{~S} \_5 \mathrm{LV}$} & \multirow{2}{*}{71} & Steel stirrups & $2 \phi 6$ with two legs $(0.10)$ & 300 & 90 \\
\hline & & CFRP strips & $2 \times 5$ strips with $1.4 \times 10 \mathrm{~mm}^{2}(0.10)$ & 160 & 90 \\
\hline $2 \mathrm{~S} \_8 \mathrm{LV}$ & 70 & Steel stirrups & $2 \phi 6$ with two legs $(0.10)$ & 300 & 90 \\
\hline 2S_3LI45 & 66 & CFRP strips & $2 \times 3$ strips with $1.4 \times 10 \mathrm{~mm}^{2}(0.06)$ & 367 & 45 \\
\hline \multirow{2}{*}{ 2S_5LI45 } & \multirow{2}{*}{64} & Steel stirrups & $2 \phi 6$ with two legs $(0.10)$ & 300 & 90 \\
\hline & & CFRP strips & $2 \times 5$ strips with $1.4 \times 10 \mathrm{~mm}^{2}(0.10)$ & 220 & 45 \\
\hline \multirow{2}{*}{ 2S_8LI45 } & \multirow{2}{*}{68} & Steel stirrups & $2 \phi 6$ with two legs $(0.10)$ & 300 & 90 \\
\hline & & CFRP strips & $2 \times 8$ strips with $1.4 \times 10 \mathrm{~mm}^{2}(0.16)$ & 138 & 45 \\
\hline \multirow{2}{*}{ 2S_3LI60 } & \multirow{2}{*}{71} & Steel stirrups & $2 \phi 6$ with two legs $(0.10)$ & 300 & 90 \\
\hline & & CFRP strips & $2 \times 3$ strips with $1.4 \times 10 \mathrm{~mm}^{2}(0.06)$ & 325 & 60 \\
\hline 2S_5LI60 & 67 & Steel stirrups & $2 \phi 6$ with two legs $(0.10)$ & 300 & 90 \\
\hline
\end{tabular}

$\tilde{n}_{s w}=\left(A_{s w} /\left(b_{w} d\right)\right) \times 100$ (stirrups ratio); $\rho_{f w}=2 . a_{f} \cdot b_{f} /\left(b_{w} \cdot s_{f} \cdot \sin \beta\right) .100$.

beam with steel stirrups $\phi 6 @ 300 \mathrm{~mm}\left(2 \mathrm{~S} \_\mathrm{R}\right.$ beam, with stirrups ratio $\left.\rho_{f w}=0.10 \%\right)$, one beam with steel stirrups $\phi 6 @ 130 \mathrm{~mm}\left(6 \mathrm{~S} \_\mathrm{R}\right.$ beam, $\left.\rho_{f w}=0.24 \%\right)$, and nine beams of \$6@300mm with different CFRP strengthening arrangements on the $L_{l}$ beam span: three different CFRP ratios $\left(\rho_{f w}\right)$ and, for each CFRP ratio, three different strips angles ( $\beta$, angle between CFRP fibers direction and beam axis, (Fig. 6) namely, $90^{\circ}, 45^{\circ}$ and $60^{\circ}$. The CFRP shear strengthening ratio $\rho_{f w}$ (see Table 2) was obtained from $\rho_{f w}=2 . a_{f} \cdot b_{f} /\left(b_{w} \cdot s_{f} \cdot \sin \beta\right) .100 \quad$ where $a_{f}=1.4 \mathrm{~mm}$ and $b_{f}=10 \mathrm{~mm}$ are the strip cross section dimensions, $b_{w}=180 \mathrm{~mm}$ is the width of the beam's web, and $s_{f}$ is the strips spacing. For the three series of beams with different strips angles, the maximum $\rho_{f w}$ in each series was evaluated to ensure that the beams presented a maximum load similar to the 6S_R reference beam, reinforced with the highest $\rho_{s w}\left(\rho_{f w}=A_{s w} /\left(b_{w} \cdot s_{w}\right) .100\right.$, where $A_{s w}$ is the cross sectional area of the two arms of a steel stirrup and $s_{w}$ is the stirrups spacing). In the evaluation of the maximum $\rho_{f w}$ it was assumed that CFRP works at a stress level corresponding to $0.5 \%$ strain, which is a compromise between the value $0.4 \%$ recommended by ACI [7] for EBR and the $0.59 \%$ value obtained in pullout bending tests on NSM bars [8]. For the intermediate and minimum $\rho_{f w}$, the spacing $s_{f}$ for beams with $\beta$ equal to $90^{\circ}, 60^{\circ}$ and $45^{\circ}$ was evaluated to obtain a similar strips contribution for each $\rho_{f w}$. With reference to (Fig. 1), the strips were distributed along the AB line, where $\mathrm{A}$ is the beam support at the "test side" and B was obtained assuming a $45^{\circ}$ load transfer. To avoid concrete spalling at A, a confinement system made from wet lay-up CFRP sheets (three layers, with fibers aligned with the beam axis) was applied, as shown in Fig. 1. The strengthening procedures are detailed elsewhere [3].

Three point beam bending tests (see Fig. 1) were carried out using a servo closed-loop control equipment, taking the signal read in the linear variable differential transducer (LVDT) placed at the loaded section to control the test at a deflection rate of $0.01 \mathrm{~mm} / \mathrm{s}$. 
Table 2. Material Properties

\begin{tabular}{|c|c|c|c|c|c|}
\hline \multirow[b]{2}{*}{ Concrete } & \multicolumn{5}{|c|}{ Compressive strength } \\
\hline & \multicolumn{2}{|c|}{$\begin{array}{l}f_{c m}=26.0 \mathrm{MPa} \\
\quad \text { (at } 28 \text { days) }\end{array}$} & \multicolumn{3}{|c|}{$\begin{array}{c}f_{c m}=31.1 \mathrm{MPa} \\
\text { (at } 70 \text { days - age of beam tests) }\end{array}$} \\
\hline \multirow{3}{*}{ Steel } & Tensile strength & $\phi 6$ & $\phi 12$ & $\phi 16$ & $\phi 25$ \\
\hline & $\mathrm{f}_{\mathrm{sym}} *$ & $533 \mathrm{MPa}$ & $446 \mathrm{MPa}$ & $447 \mathrm{MPa}$ & $444 \mathrm{MPa}$ \\
\hline & $\mathrm{f}_{\mathrm{sum}} * *$ & $592 \mathrm{MPa}$ & $564 \mathrm{MPa}$ & $561 \mathrm{MPa}$ & $574 \mathrm{MPa}$ \\
\hline \multirow{2}{*}{ CFRP strips } & Tensile strength & \multicolumn{2}{|c|}{ Young's Modulus } & Maximum strain $* * *$ & Thickness \\
\hline & $f_{\text {fum }}=2952 \mathrm{MPa} * *$ & \multicolumn{2}{|c|}{$E_{f m}=166.6 \mathrm{GPa}$} & $\varepsilon_{\text {fum }}=1.77 \%$ & $1.4 \mathrm{~mm}$ \\
\hline
\end{tabular}

* Mean value of the yield stress; ** Mean value of the maximum stress; *** Obtained from Hooke's law.

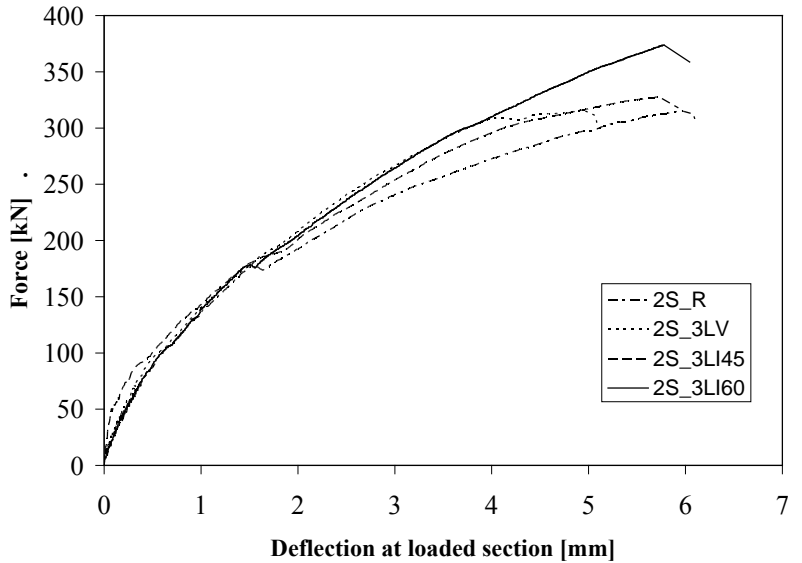

(a)

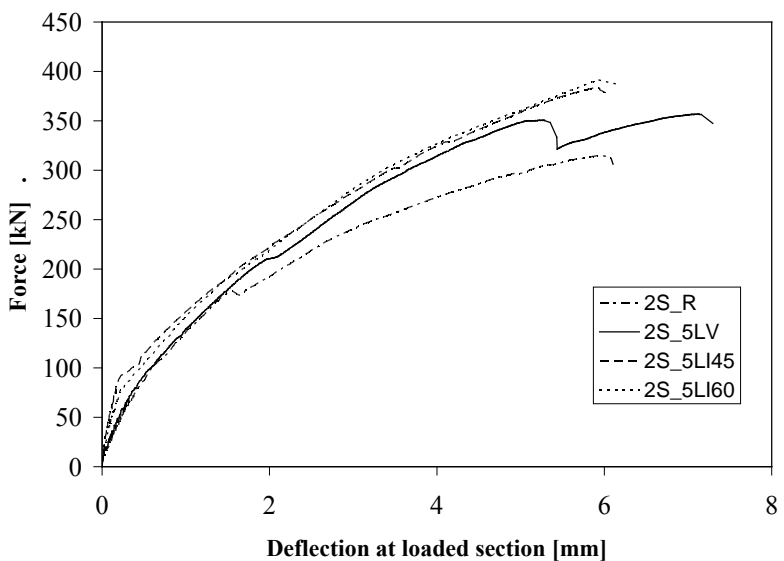

(b)

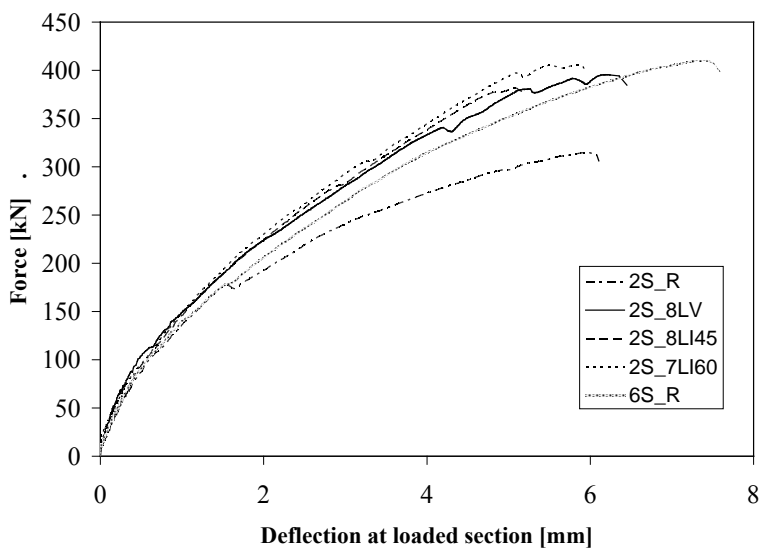

(c)

Fig. (3). Force vs. deflection at the loaded-section of the beams strengthened with: (a) minimum; (b) intermediate and (c) maximum CFRP shear strengthening ratio.

The concrete compressive strength was evaluated at 28 days and at the age of the beam test, carrying out direct compression tests on cylinders of $150 \mathrm{~mm}$ diameter and $300 \mathrm{~mm}$ height, according to EN 206-1 Standard [9]. Deformed steel bars of $6,12,16$ and $25 \mathrm{~mm}$ diameter were used in the tested beams. The main properties were obtained from uni-axial tensile tests performed according to the recommendations of EN 10002 [10]. The tensile properties of the $S \& P ®$ strips, CFK 150/2000, were characterized by uni-axial tensile tests carried out according to ISO 527-5 [11]. These strips had a cross section of $10 \times 1.4 \mathrm{~mm}^{2}$. Table 2 lists the mean values obtained from these experimental tests.

\section{Main Results and Discussion}

Table 3 includes the values of the $\Delta F_{\max } / F_{\max }^{2 S-R}$ and $F_{\max } / F_{\max }^{6 S-R}$ ratios, where $\Delta F_{\max }=F_{\max }-F_{\max }^{2 S-R}$, and $F_{\max }, F_{\max }^{2 S-R}$ and $F_{\max }^{6 S-R}$ represent, respectively, the load carrying capacity of a tested beam, of the $2 \mathrm{~S} \_\mathrm{R}$ and of the 6S_R reference beams.

The force-deflection relationships at the loading point of the tested beams are depicted in Fig. (3). If $F_{\max }^{2 S-R}$ is used as a basis of comparison, Table 3 and Fig. (3) show that, apart 
Table 3. Summary of Relevant Results of the Tested Beams

\begin{tabular}{|c|c|c|c|}
\hline $\begin{array}{c}\text { Beam } \\
\text { label }\end{array}$ & $\begin{array}{c}F_{\text {max }} \\
{[\mathrm{kN}]}\end{array}$ & $\begin{array}{c}\Delta F_{\max } / F_{\max }^{2 S-R} \\
{[\%]}\end{array}$ & $F_{\text {max }} / F_{\text {max }}^{6 S-R}$ \\
\hline \hline C_R & 243 & - & 0.59 \\
\hline 2S_R & 315 & 0.0 & 0.77 \\
\hline 6S_R & 410 & 30.2 & 1.00 \\
\hline 2S_3LV & 316 & 0.3 & 0.77 \\
\hline 2S_5LV & 357 & 13.3 & 0.87 \\
\hline 2S_8LV & 396 & 25.7 & 0.97 \\
\hline 2S_3LI45 & 328 & 4.1 & 0.80 \\
\hline 2S_5LI45 & 384 & 21.9 & 0.94 \\
\hline 2S_8LI45 & 382 & 21.3 & 0.93 \\
\hline 2S_3LI60 & 374 & 18.7 & 0.91 \\
\hline 2S_5LI60 & 392 & 24.4 & 0.96 \\
\hline 2S_7LI60 & 406 & 28.9 & 0.99 \\
\hline
\end{tabular}

from the $2 \mathrm{~S} \_3 \mathrm{LV}$ beam, all adopted CFRP strengthening configurations provided an increase in the beam load carrying capacity, for any $\rho_{f w}$ and $\beta$. The load decay observed in the 2S_R reference beam, when a shear crack formed, did not occur in CFRP shear strengthened beams, revealing that strips delayed the formation of the shear failure crack. The strengthening arrangements with the lowest $\rho_{f w}$ presented the smaller increments in terms of beam load carrying capacity: $0.3 \%, 4.1 \%$ and $18.7 \%$ for the beams strengthened with strips at $90^{\circ}, 45^{\circ}$ and $60^{\circ}$, respectively, see Fig. 3a. However, the increment in the beam load capacity that these strengthening systems provided for deflections above the one corresponding to the formation of the shear failure crack in the $2 \mathrm{~S} \_\mathrm{R}$ reference beam was appreciable, even for $2 \mathrm{~S} \_3 \mathrm{LV}$ beam.
The strengthening configurations of strips at $90^{\circ}, 45^{\circ}$, and $60^{\circ}$, for intermediate $\tilde{n}_{f w}$, provided an increase in the maximum load of $13.3 \%, 21.9 \%$ and $24.4 \%$, respectively (see Fig. 3b and Table 3). Amongst the beams strengthened with the highest $\tilde{n}_{f w}$, the strengthening configuration with $\beta=60^{\circ}$ was the most effective in terms of peak load: a $28.9 \%$ increase was obtained, while increments of $25.7 \%$ and $21.3 \%$ were recorded for $\beta=90^{\circ}$ and $\beta=45^{\circ}$, respectively.

As mentioned above, the highest $\tilde{n}_{f w}$ for each strengthening arrangement was designed to achieve a peak load close to that of the $6 \mathrm{~S} \_\mathrm{R}$ reference beam. The obtained experimental results show that, in general, this was attained, since the maximum load of the beams with $\beta=90^{\circ}, 45^{\circ}$ and $60^{\circ}$ reached $97 \%, 93 \%$ and $99 \%$, respectively, of the maximum load of the 6S_R reference beam (see Fig. 3c and Table 3). The most notable aspect is, however, the larger load-carrying capacity of the strengthened beams with respect to the $6 \mathrm{~S} \_\mathrm{R}$ reference beam, after shear crack initiation of the $2 \mathrm{~S} \_\mathrm{R}$ beam (see Fig.3c). This improved performance of the strengthened beams can be ascribed to the stiffness contribution provided by the strips.

It is worth pointing out that in the beams strengthened with higher strips' shear strengthening ratio, a layer of concrete, approximately as thick as the cover, and containing the glued strips, progressively detached from the core of the beam web (see Figs. $\mathbf{4 e}$ and $\mathbf{4 f}$ ).

Moreover, the effective strain, $\varepsilon_{f}^{\exp }$, i.e. the average of the strains recorded along the monitored strip for each beam, presented a general tendency to increase by increasing the spacing between strips, $s_{f}^{\prime}$, measured orthogonally to their inclination (see Fig. 5). Further details about both the ob-

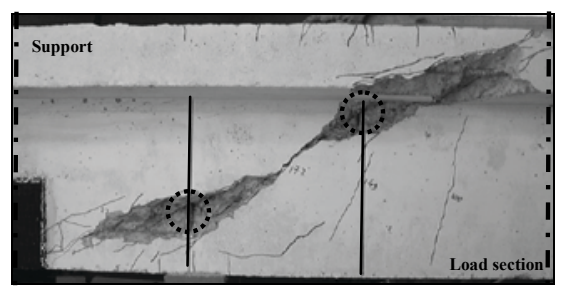

(a) $2 \mathrm{~S} \mathrm{R}$

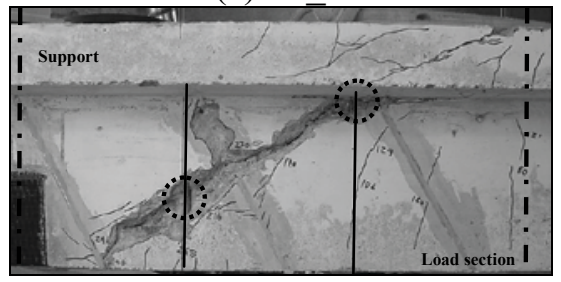

(d) 2S_3LI60

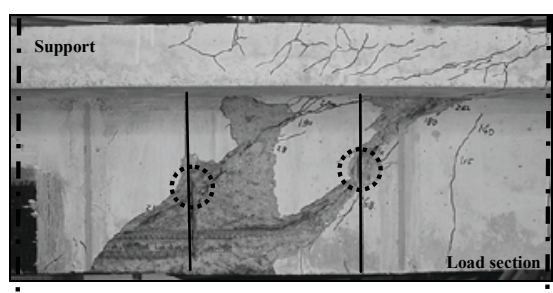

(b) $2 \mathrm{~S} \_3 \mathrm{LV}$

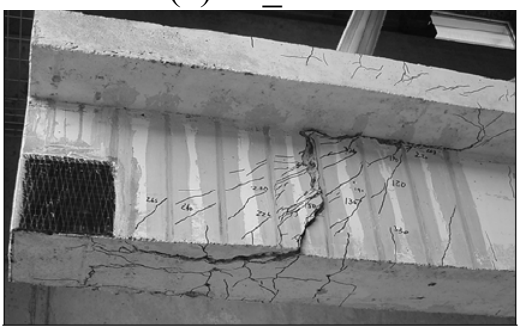

(e) $2 \mathrm{~S} \_8 \mathrm{LV}$

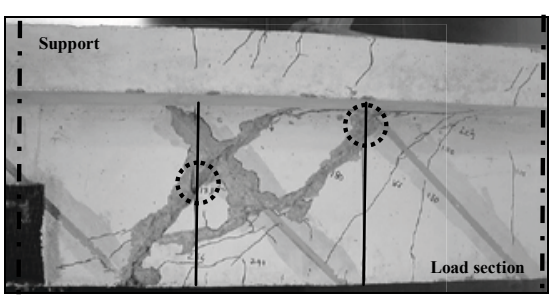

(c) $2 \mathrm{~S} 3$ LI45

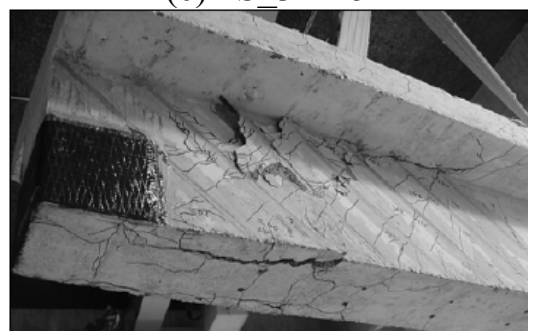

(f) 2S_8LI45

Fig. (4). Some details of the failure zones: beam (a) 2S_R; (b) 2S_3LV; (c) 2S_3LI45; (d) 2S_3LI60. And observed failure mechanisms: beams (e) $2 \mathrm{~S} \_8 \mathrm{LV}$; (f) 2S_8LI45. 


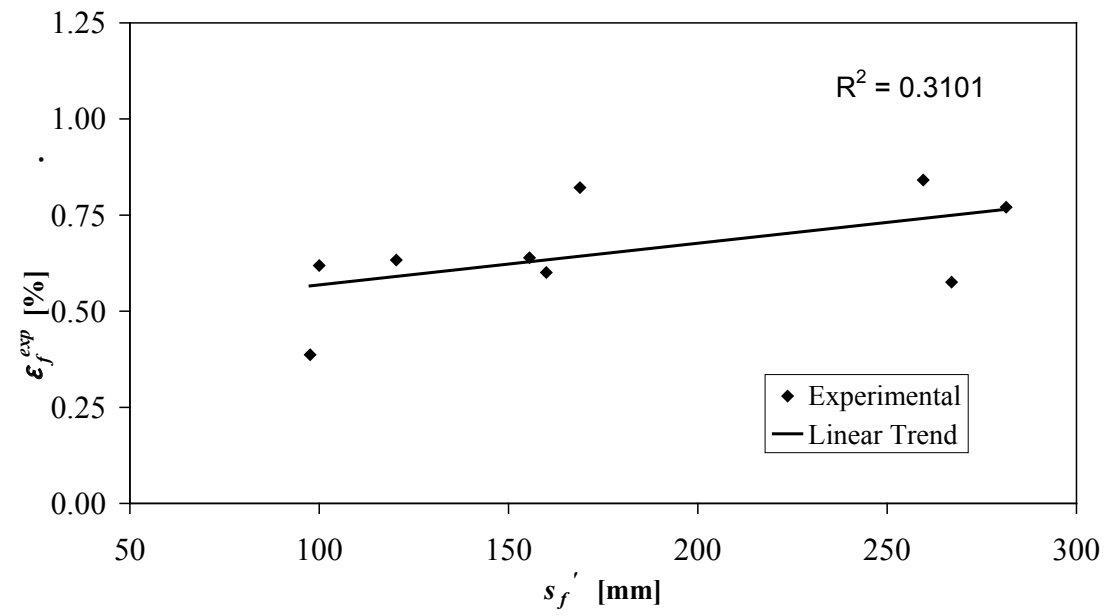

Fig. (5). Influence of the CFRP percentage on the recorded effective strain.
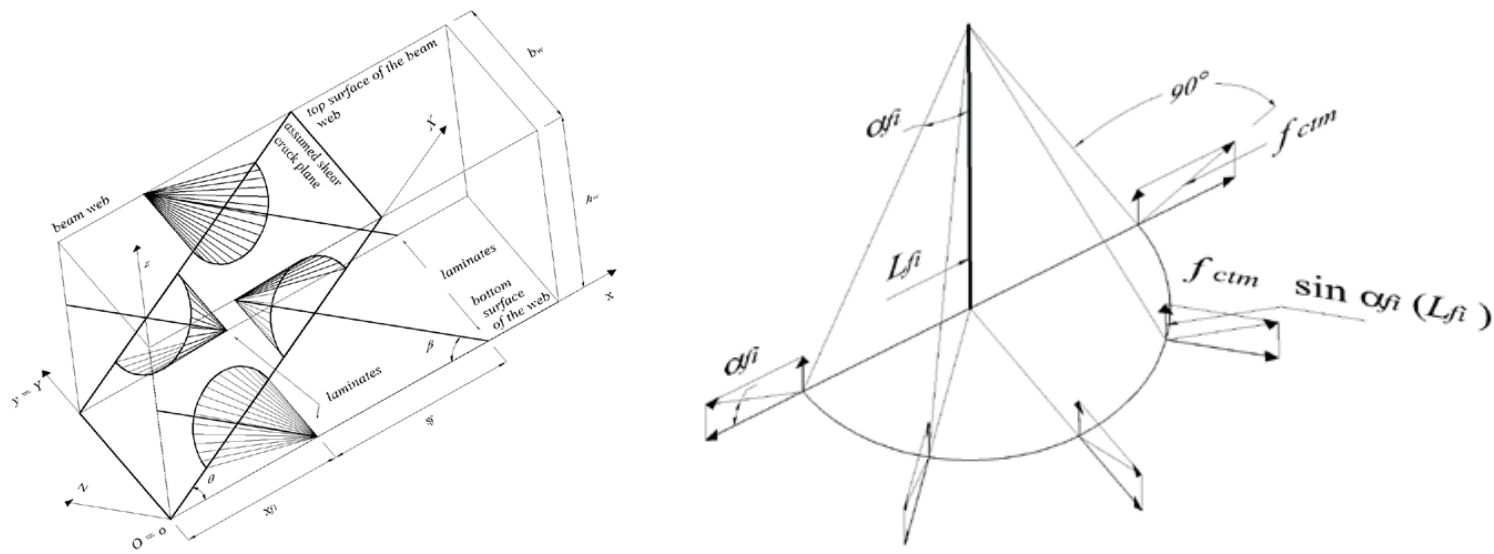

Fig. (6). Main features of the proposed model: a) crack plane crossed by strips and their semi-conical fracture surfaces; b) detail of the semiconical fracture surface and the distribution of the average tensile strength.

served failure modes and the strains measured in the monitored steel stirrups and CFRP strips can be found elsewhere $[6,12]$.

\section{NEW MODEL}

\section{Model Physical Fundamentals}

By searching the technical literature available to date, the analogy arises between the NSM technique and the fastening technology to concrete by means of adhesive anchors [1316]. This latter consists in fixing anchors into holes drilled in the soffit of whatever RC structure by different kinds of structural adhesives. As for the NSM strips, the stress transfer of anchors strongly relies on the bond characteristics. The experimental evidence in the field of fastening technology reported three possible failure modes: tensile rupture of the anchor, debonding and another failure mode designated as "concrete cone failure" [14, 15]. This latter is characterized by a cone-shaped spalling of the concrete surrounding the anchor originating at a certain point of the embedded length of the anchor and propagating towards the external surface of the concrete [5]. This failure occurs when the applied force is such as to induce, in the surrounding concrete, principal stresses exceeding its tensile strength. The resulting concrete fracture conical surface, envelope of the tension isostatics, shows, at its vertex, an angle of about $45^{\circ}$ with the anchor axis.

In the case of NSM strips, the critical diagonal crack can be schematized like a plane slicing the web of the beam in two parts sewn together by the crossing strips that can be regarded as fastenings (see Fig. 6a). The strips may fail along their "available bond length" (is the shorter length on either side of the crossing crack [5]) by: debonding, tensile rupture or concrete tensile fracture. The different and asymmetric geometrical features support the assumption that, in the case of the strips glued into thin slits in the concrete web face, the concrete fracture surface, envelope of the principal tensile stresses induced in the surrounding concrete, has a semi-conical shape propagating from the inner tip of the strip embedded length. The concrete average tensile strength, $f_{c t m}$, is distributed throughout each of the resulting semiconical surfaces orthogonally to them in each point (see Fig. 6b).

The NSM shear strength contribution, $V_{f}$, can be calculated by adding the contribution ascribed to each strip, $V_{f i}^{p}$, parallel to its orientation, and projecting the resulting force orthogonally to the beam axis, according to the following formula: 


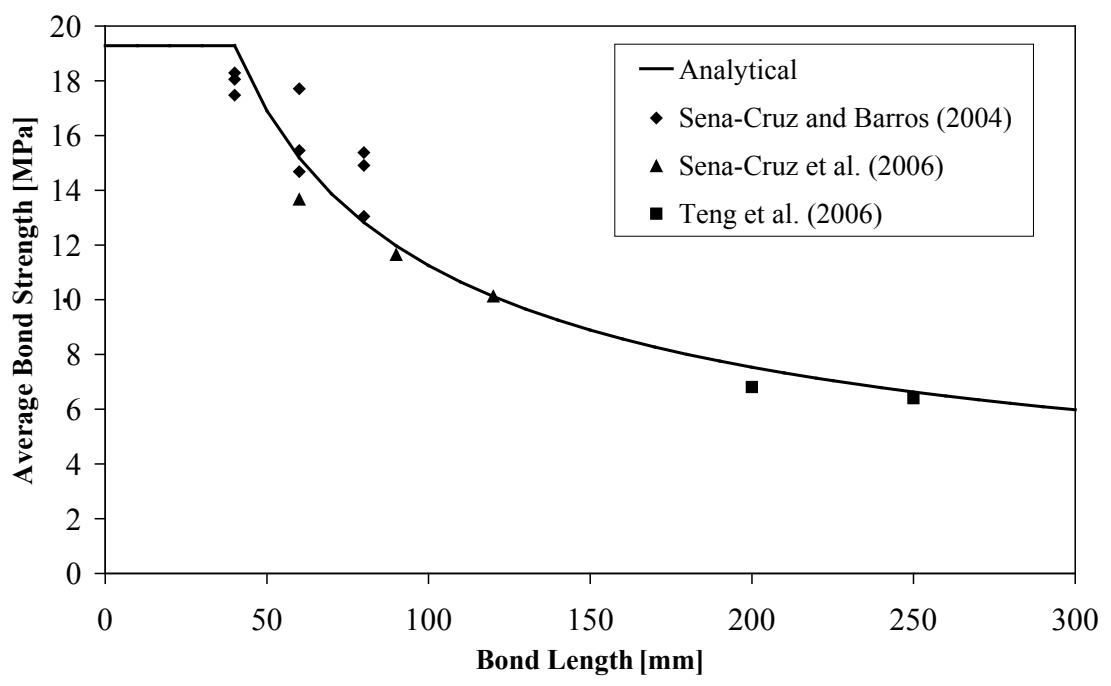

Fig. (7). Average bond strength vs. bonded length $[19,20]$.

$$
V_{f}=2 \cdot \sin \beta \cdot \sum_{i=1}^{N_{f}} V_{f i}^{p}
$$

where $N_{f}$ is the number of the strips crossing the shear crack. The contribution provided by each strip, $V_{f i}^{p}$, can be assumed as the minimum among the three possible contributions ascribed respectively to debonding, $V_{f i}^{p, d b}$, tensile rupture of the strip, $V_{f i}^{p, t r}$, or concrete tensile fracture, $V_{f i}^{p, c f}$, i.e.:

$$
V_{f i}^{p}=\min \left\{V_{f i}^{p, d b} ; V_{f i}^{p, t r} ; V_{f i}^{p, c f}\right\}
$$

The debonding-based term, $V_{f i}^{p, d b}$, ascribed to the i-th strip and parallel to its orientation can be computed like follows:

$$
V_{f i}^{p, d b}=2 \cdot\left(a_{f}+b_{f}\right) \cdot \tau_{b}\left(L_{f}\right) \cdot L_{f}
$$

where $\tau_{b}\left(L_{f}\right)$ is the length-dependent value of the average bond strength. The adopted relationship between average bond strength (in $\mathrm{MPa}$ ) and bond length (in $\mathrm{mm}$ ) is the following (Fig. 7) [17, 18]:

$$
\tau_{b}\left(L_{f}\right)=\left\{\begin{array}{lr}
19.28 & 0<L_{f}<40 \\
0.355+174.613 .\left(L_{f}\right)^{-0.60233} & L_{f} \geq 40
\end{array}\right.
$$

The tensile rupture-based term, $V_{f i}^{p, t r}$, ascribed to each strip and parallel to its orientation is equal to:

$$
V_{f i}^{p, t r}=a_{f} \cdot b_{f} \cdot f_{f u}
$$

where $f_{f u}$ is the tensile strength of the adopted CFRP strips.

The concrete fracture-based term, $V_{f i}^{p, c f}$, ascribed to each strip and parallel to its orientation, can be calculated distributing the component of the concrete average tensile strength parallel to the strip, i.e., $f_{c t m} \sin \alpha_{f i}$, throughout the resulting relevant semi-conical surface and integrating, according to the following formula (Fig. 6b):

$$
V_{f i}^{p, c f}=\int_{C_{f i}\left(L_{f i} ; \alpha_{f i}\right)}\left(f_{c t m} \cdot \sin \alpha_{f i}\right) \cdot d C_{f i}
$$

where $C_{f i}\left(L_{f i} ; \alpha_{f i}\right)$ concisely denotes the semi-conical surface associated to the i-th strip and $\alpha_{f i}$ is the angle between the generatrices and the axis of the semi-cone attributed to the i-th strip.

The angle between the axis of the semi-conical surface and its generatrices, $\alpha_{f}$, calibrated on the basis of the interpretation of some experimental results available to date [5, 18], ranges approximately between $20^{\circ}$ and $30^{\circ}$ and shows a length-dependency on the available bond length, $L_{f}$, but, in this respect, further investigations are required. The relationship between the angle, $\alpha_{f i}$ (in degrees), and the available bond length, $L_{f i}$ (in $\mathrm{mm}$ ), assumed in the present work is the following:

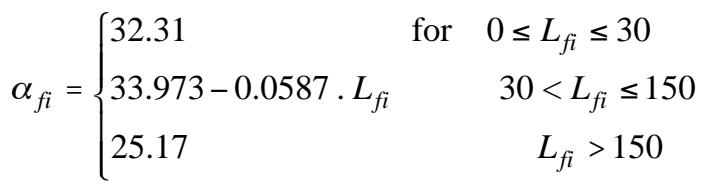

Further details can be found elsewhere [5]. If attention is focused on one strip only, in the case in which it results to be orthogonal to the crack plane and in complete absence of interaction with the contiguous ones, the shear strength contribution parallel to its orientation $V_{f i}^{p}$ can be calculated by: 


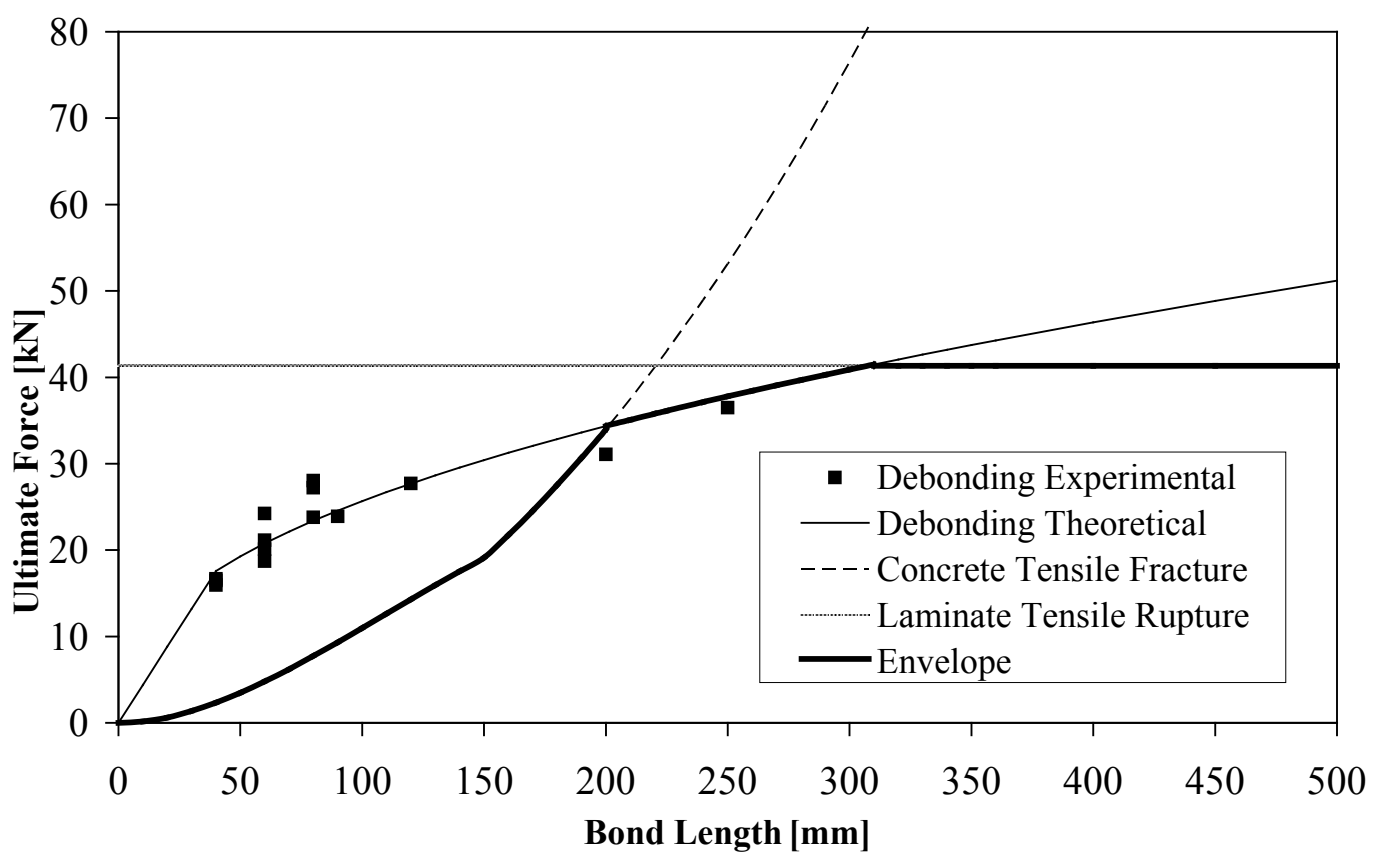

Fig. (8). Expected failure mode as function of the available bond length.

$$
V_{f i}^{p}=\min \left\{2 \cdot\left(a_{f}+b_{f}\right) \tau_{b}\left(L_{f i}\right) \cdot L_{f i} ; a_{f} \cdot b_{f} \cdot f_{f u} ;\left(\frac{\pi}{2} \cdot f_{c t m}\right) \cdot \operatorname{tg}^{2} \alpha_{f i} \cdot L_{f i}^{2}\right\}
$$

that, for the materials regarding the experimental program presented in the companion paper, is plotted in Fig. 8. It arises that: for a value of the available bond length up to $200 \mathrm{~mm}$ the prevailing failure mode is the concrete semi-conical fracture; for a value between 200 and $310 \mathrm{~mm}$ the failure mode is debonding, and for an available bond length higher than $310 \mathrm{~mm}$ the strips are expected to fail by tensile rupture. Due to the interaction between contiguous strips, the curve regarding the concrete tensile fracture opens downwards or upwards (when strips spacing decreases and increases, respectively) thus changing the range of length values in correspondence of which debonding is expected to be the commanding failure mode. The terms $V_{f i}^{p, t r}$ and $V_{f i}^{p, d b}$, based on the phenomenon of tensile rupture and debonding of the strip, respectively, are intrinsically independent of the interaction between subsequent strips that, on the contrary, affects the concrete fracture-based term, $V_{f i}^{p, c f}$.

As the spacing between subsequent strips is reduced, their semi-conical fracture surfaces overlap and the resulting envelope area progressively becomes smaller than the mere summation of each of them (see Fig. 9a). This detrimental interaction between strips can be easily taken into account by calculating the resulting semi-conical surface ascribed to each strip accordingly. For very short values of the spacing, the resulting concrete failure surface is almost parallel to the web face of the beam, which is in agreement with the failure mode observed experimentally, consisting in the detachment of the concrete cover from the underlying core of the beam (see Figs. 4e and 4f). Since the position of those semiconical surfaces is symmetric with respect to the vertical plane passing through the beam axis, the horizontal outward components of the tensile strength vectors distributed throughout their surfaces are balanced only from an overall standpoint but not locally (see Fig. 9b). This local unbalance of the horizontal tensile stress component orthogonal to the beam web face justifies the outward expulsion of the concrete cover in both the uppermost and lowermost parts of the strengthened sides of the web. The post-test photographic documentation (see Figs. 4e and $\mathbf{4 f}$ ) clearly spotlights this local occurrence.

\section{Analytical Formulation}

In Eq. (6), the operation of integrating the component of the concrete tensile strength parallel to the strip, $f_{c t m} \sin \alpha_{f i}$, throughout the relevant semi-conical surface is equivalent to projecting the surface on a plane orthogonal to the strip and multiplying it by the absolute value of the concrete average tensile strength [11] i.e.:

$$
d \mathrm{~A}_{f i}=d C_{f i} \cdot \sin \alpha_{f i}
$$

Introducing (9.1) in (6) results:

$V_{f i}^{p, c f}=\int_{C_{f i}\left(L_{f i} ; \alpha_{f i}\right)}\left(d C_{f i} \cdot \sin \alpha_{f i}\right) \cdot f_{c t m}=f_{c t m} \cdot \int_{\mathrm{A}_{f i}\left(L_{f i} ; \alpha_{f i}\right)} d \mathrm{~A}_{f i}=f_{c t m} \cdot \mathrm{A}_{f i}\left(L_{f i} ; \alpha_{f i}\right)$

where $\mathrm{A}_{f i}\left(L_{f i} ; \alpha_{f i}\right)$ is the area, function of both the available bond length $L_{f i}$ and the angle $\alpha_{f i}$, obtained by projecting the semi-conical surface on a plane orthogonal to the strip (see Fig. 10).

Since the intersection of each semi-conical surface with the crack plane is constituted by a semi-ellipse, that becomes a semi-circle in the particular case in which the strip is or- 


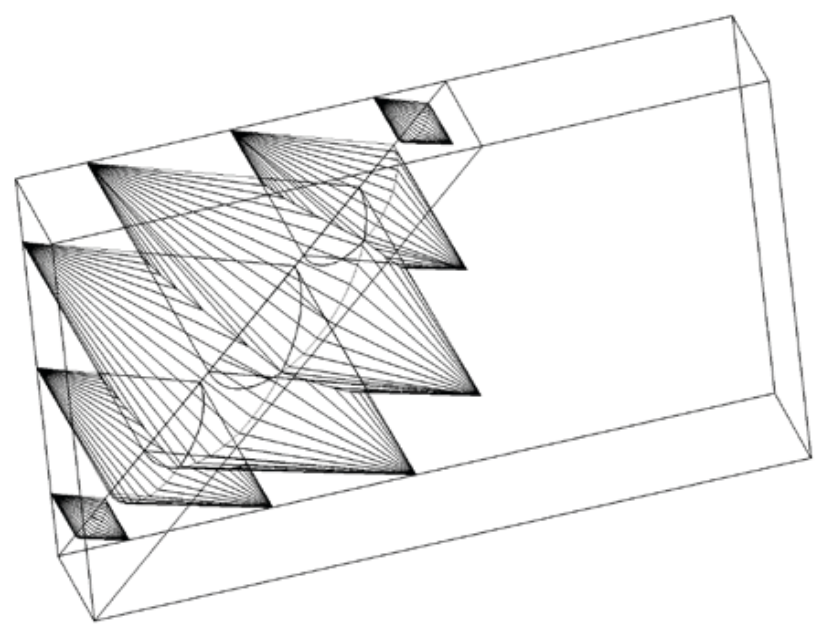

a)
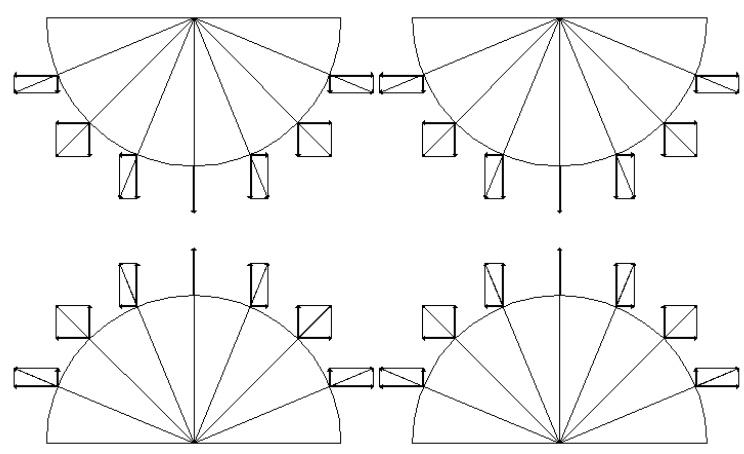

b)

Fig. (9). Interaction between strips and outward expulsion of the strengthened concrete cover: a) inside view of the fracture surface resulting from the overlapping of semi-conical fracture surfaces on one side of the web; b) local unbalance of the components of the concrete tensile strength orthogonal to the web faces on a section parallel to the crack plane.

thogonal to the crack plane, the above area $\mathrm{A}_{f i}\left(L_{f i} ; \alpha_{f i}\right)$ can be evaluated by calculating the area of the semi-ellipse and then projecting this latter on the plane orthogonal to the strip (see Fig. 10). Hence, the main point of the calculation of the contribution ascribed to the i-th strip parallel to its length, $V_{f i}^{p, c f}$, is reduced to the evaluation of the area underlying the relevant semi ellipse, i.e.:

$$
V_{f i}^{p, c f}=\sin (\theta+\beta) \cdot f_{c t m} \int_{E_{f i}\left(L_{f i} ; \alpha_{f i}\right)} d E_{f i}
$$

where $E_{f i}\left(L_{f i} ; \alpha_{f i}\right)$ is the equation of the semi-ellipse, intersection of the $i$-th semi-conical surface with the assumed shear crack plane. This simplification is extremely powerful from a computational standpoint since allows the interaction between strips to be easily accounted for. In function of the main geometrical parameters $h_{w}, b_{w}, s_{f}, L_{f i}$ and $\alpha_{f i}\left(L_{f i}\right)$, see Fig. 6, that interaction can be either monodirectional, longitudinal or transversal, or bi-directional. The longitudinal interaction can occur when, due to the reduced spacing with respect to the height of the web, the semi-cones associated to adjacent strips located at the same side of the web, and consequently their relevant semi-ellipses, overlap along their major semi-axis (see for instance the semiellipses 5 and 6 of the example of Fig. 11). The transversal interaction can occur when, for slender beam cross sections of high $h_{w} / b_{w}$ ratio, the semi-ellipses symmetrically placed on the opposite sides of the web, intersect each other along their minor semi-axis (see the semi-ellipse 1 of Fig. 11). In

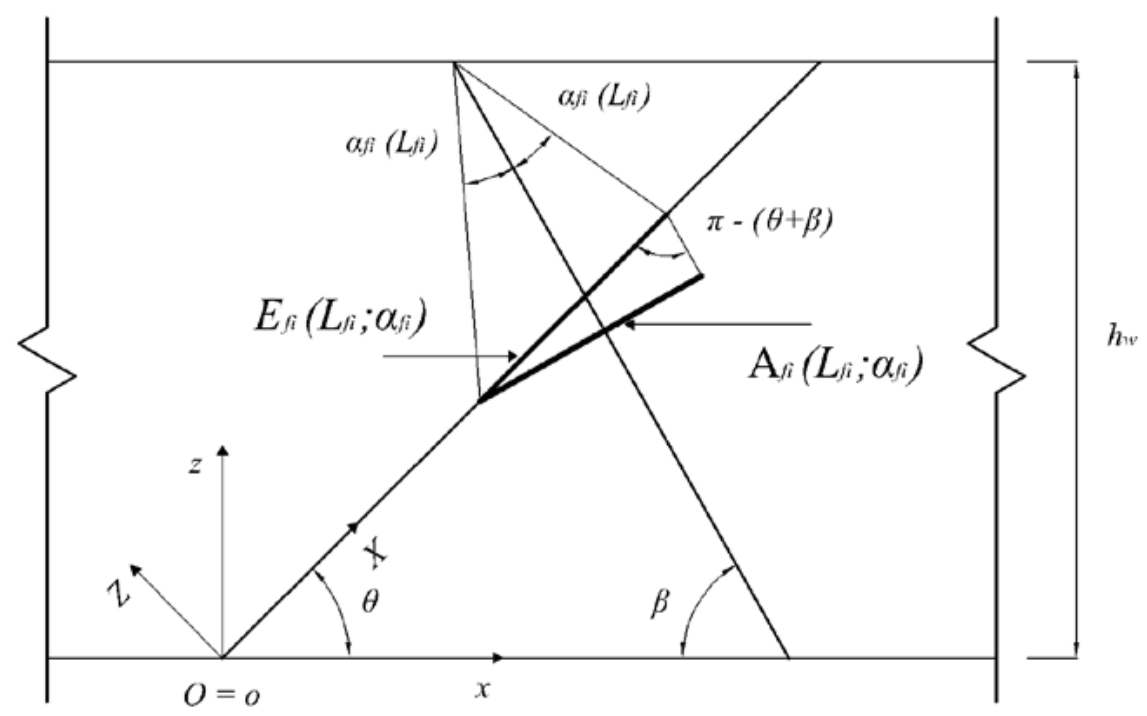

Fig. (10). Projection of the semi-conical surface on a plane orthogonal to the strip. 


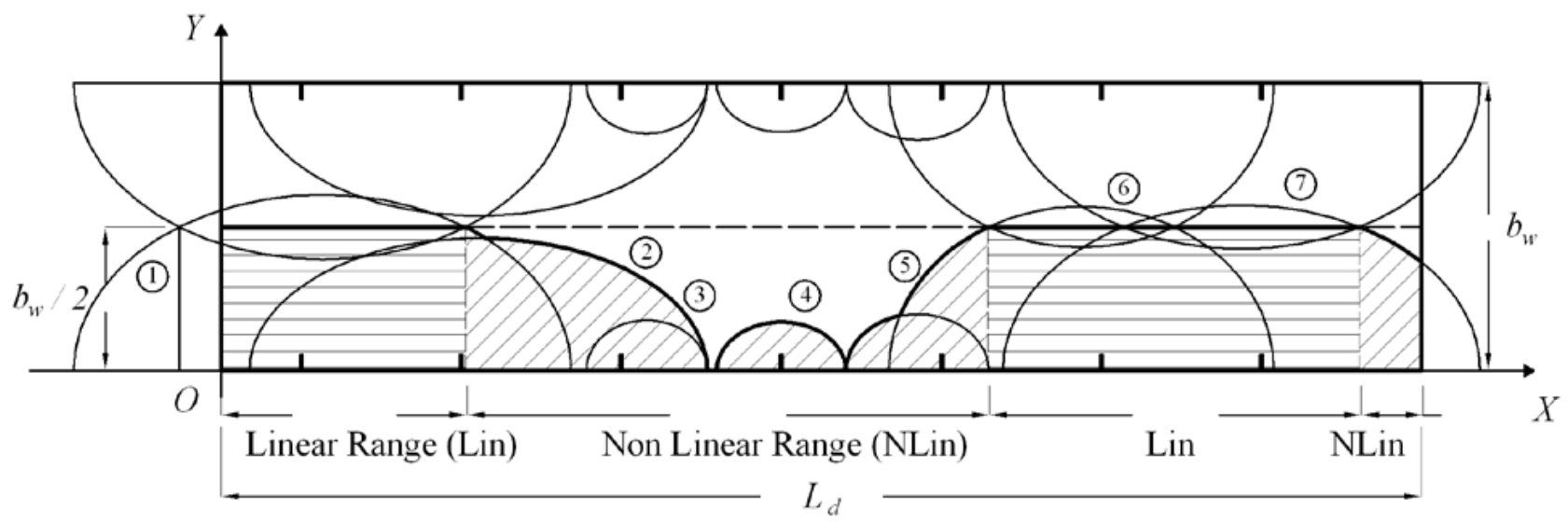

Fig. (11). Definition of half crack plane and linear and non-linear range of integration for each ellipse.

this latter case, the area of the i-th semi-ellipse is limited, upwards, by the line $Y=b_{w} / 2$, i.e. the trace, on the shear crack plane, of the vertical plane passing through the beam axis. In the most general case, in which bidirectional interaction might occur, the area on the shear crack plane associated to the i-th strip, would be composed of two terms: one, $\mathrm{A}_{f i}^{\text {nlin }}$, limited upwards by the non-linear branch of the relevant semi-ellipse $Y_{i}(X)$ and another, $\mathrm{A}_{f i}^{l i n}$, limited by the line $Y=b_{w} / 2$ (see the semi-ellipses 1,6 and 7 of Fig. 11). Hence, due to the bi-directional interaction, the area of the semi-ellipse associated to the i-th strip is calculated as follows:

$\int_{E_{f i}\left(L_{f i} ; \alpha_{f i}\right)} d E_{f i}=\left(\mathrm{A}_{f i}^{n l i n}+\mathrm{A}_{f i}^{l i n}\right)$

Ultimately, the equation (1) can be re-written as follows:
- $h_{w}$, the height of the web in the case of a T cross section beam. For a rectangular cross section beam, $h_{w}$ is the vertical component of the strip length, i.e., $h_{w}=L_{f} / \sin \beta$, where $L_{f}$ is the strip length;

- $b_{w}$, the width of the web of the beam cross section in the case of a $\mathrm{T}$ beam. For a rectangular cross section beam, $b_{w}$ is the cross section width;

- $\beta$, the inclination of the strips with respect to the beam axis;

- $s_{f}$, the spacing of the strips along the beam axis;

- $\theta$, the assumed crack angle;

- $\alpha_{f i}\left(L_{f i}\right)$, the relationship between the angle, formed by the axis and the generatrices of the i-th semiconical surface, and the available bond length of the strip;

- $\quad f_{c t m}$, the concrete average tensile strength;

$$
V_{f}=2 \cdot \sin \beta \cdot \sum_{i=1}^{N_{f}} \min \left\{2 \cdot\left(a_{f}+b_{f}\right) \cdot L_{f i} \cdot \tau_{b}\left(L_{f i}\right) ; a_{f} \cdot b_{f} \cdot f_{f u} ;\left(\mathrm{A}_{i}^{n l i n}+\mathrm{A}_{i}^{l i n}\right) \cdot \sin (\theta+\beta) \cdot f_{c t m}\right\}
$$

In the following, the model is developed taking into consideration the three geometrical configurations, for $k=1,2,3$ (see Fig. 12). Three different configurations of the strips with respect to the assumed crack origin are considered in order to get a general approach for the relative position between the shear failure crack and the intersected strips. More details can be found elsewhere [5].

The configuration is reflected by the digit after comma present in the subscript of each configuration-dependent quantity.

\section{Input Data}

The input parameters taking part in the developed analytical model are the following (see Fig. 6):
- $a_{f}$, the thickness of the strip cross section;

- $b_{f}$, the width of the strip cross section;

- $f_{f u}$, the strip tensile strength;

- $\tau_{b}\left(L_{f}\right)$ relationship between the average bond strength and the available bond length of the strip.

- The formulation requires the use of the following two Cartesian reference systems (see Fig. 6):

- oxyz global reference system whose origin is placed in the assumed crack origin and whose plane oxy lies on the intrados of the prism schematizing the beam web; 


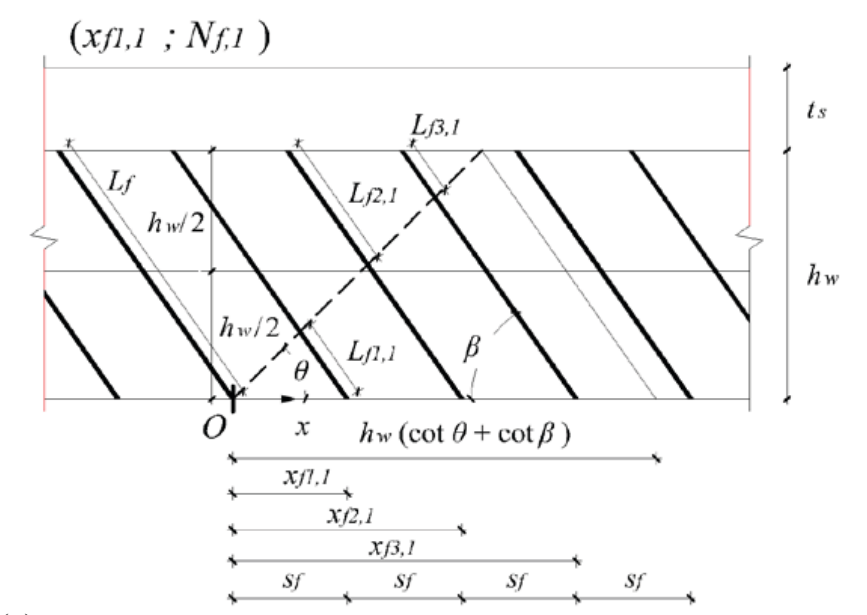

(a)

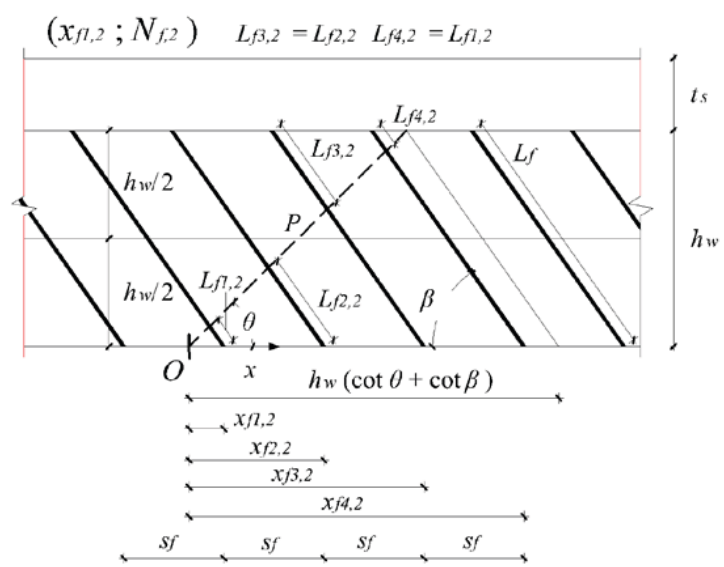

(b)

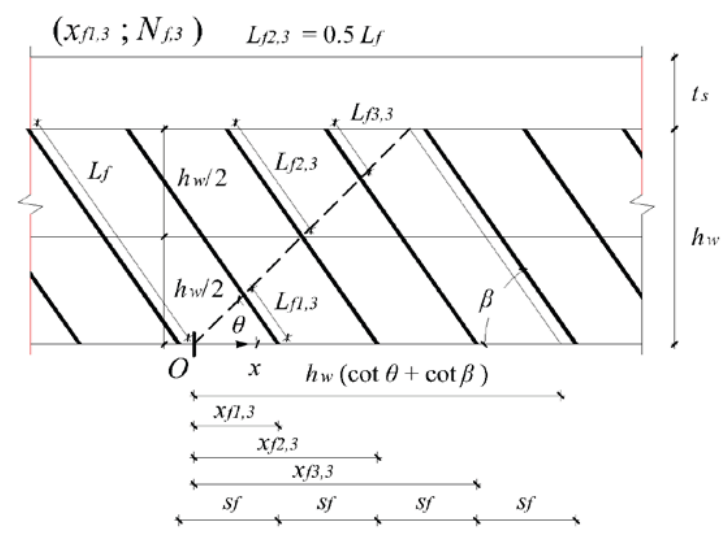

(c)

Fig. (12). The (a) first, (b) second and (c) third considered configurations for the strips [5].

- $O X Y Z$ the crack plane reference system whose origin is placed in the assumed crack origin and whose plane $O X Y$ lies on the plane schematizing the crack.

\section{Definition of the Geometric Quantities in oxyz}

The output of this block of calculation is composed of two matrices summarizing the prominent geometrical quantities defined in the global reference system:
- $x$ is a $3 \times 2$ dimension matrix, the first column of which stores the position of the first strip with respect to the assumed crack origin, for the three possible strips' configurations, $x_{f 1, k}$, see Fig. 12, while the second column includes the corresponding number of strips crossing the shear failure crack, $N_{f, k}$;

$\underline{F}$ is a $N_{f} \times 3$ dimension matrix. For a generic k-th configuration, the first column of $\underline{F}_{k}$ includes the position of the strips $x_{f i, k}$, the second column stores the available bond length of the strips, $L_{f i, k}$, and the third column includes the values of the angle $\alpha_{f i, k}$. In the present model, the $i$ char in the subscript of any symbol refers the i-th strip and its associated semi-ellipse. For the generic k-th configuration it is $i=1, \ldots \ldots, N_{f, k}$.

The pair $\left(x_{f 1, k} ; N_{f, k}\right)$ can assume the following values, as function of $k=1,2,3$ :

$$
\left(x_{f 1, k} ; N_{f, k}\right)=\left\{\begin{array}{l}
{\left[s_{f} ; N_{f, \text { int }}^{l}\right]} \\
{\left[\frac{L_{f}}{2} \cdot \frac{\sin (\theta+\beta)}{\sin \theta}-\frac{\left(N_{f, e v}-1\right)}{2} \cdot s_{f} ; N_{f, e v}\right]} \\
{\left[\frac{h_{w}}{2} \cdot(\cot \theta+\cot \beta)-\frac{\left(N_{f, o d d}-1\right)}{2} \cdot s_{f} ; N_{f, o d d}\right]}
\end{array}\right.
$$

The above three pairs include, respectively: the possibility for the strips to attain the minimum total available bond length (Fig. 12a); the possibility that an even number of strips be disposed symmetrically with respect to the intersection point between the longitudinal axis of the beam's web and the shear crack plan (point $\mathrm{P}$ in Fig. 12b); the case in which one strip has the maximum length i.e., it intersects the crack at its mid-length (Fig. 12c).

The position of each strip along the assumed $x$-axis is (see Fig. 12):

$x_{f i, k}=x_{f 1, k}+(i-1) . s_{f} \quad$ for $\quad i=1 ; \ldots \ldots ; N_{f, k}$

and its available bond length, i.e. the shorter length on either side of the crossing crack, is obtained by:

$L_{f i, k}=\left\{\begin{array}{llrl}{\left[x_{f 1, k}+(i-1) \cdot s_{f}\right] \cdot \frac{\sin \theta}{\sin (\theta+\beta)}} & \text { for } & x_{f i, k}<\frac{h_{w}}{2} \cdot(\cot \theta+\cot \beta) \\ L_{f}-\left[x_{f 1, k}+(i-1) \cdot s_{f}\right] \cdot \frac{\sin \theta}{\sin (\theta+\beta)} & \text { for } & x_{f i, k} \geq \frac{h_{w}}{2} \cdot(\cot \theta+\cot \beta)\end{array}\right.$

\section{Definition of the Geometric Quantities in $O X Y Z$}

To easily determine the equations of the semi-ellipses in the crack plane reference system, the prominent geometrical quantities, for each i-th strip, are stored in the corresponding i-th row of the $\underline{G}_{k}$ matrix, that is, the $\underline{G}$ matrix in the k-th configuration, of $N_{f, k} \times 8$ dimensions. The first column of 


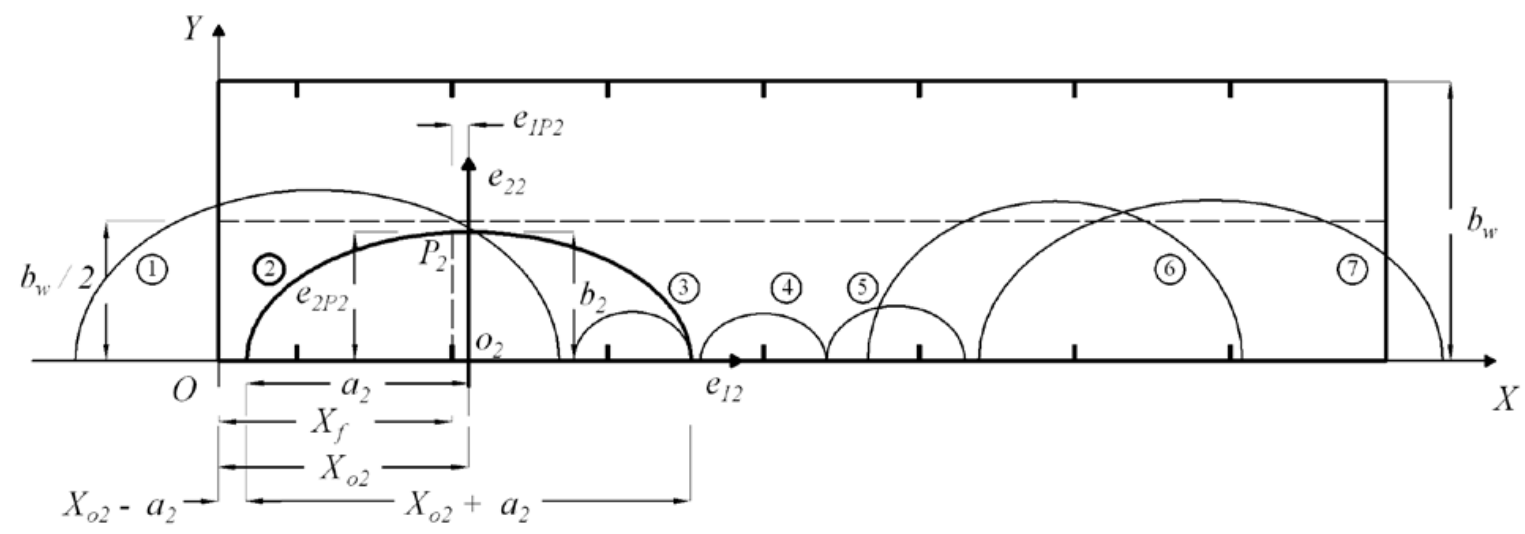

Fig. (13). Definition of the geometrical quantities in $O X Y$ and the ellipse local reference system $o_{\mathrm{i}} e_{1} i e_{2 \mathrm{i}}$.

the $\underline{G}_{k}$ matrix has the position of each strip singled out along the $O X$ axis of the crack plane reference system, $X_{f i}$ (see Fig. 13). For a generic i-th strip, $X_{f i, k}$ can be evaluated by:

$$
X_{f i, k}=\frac{\sin \beta}{\sin (\beta+\theta)} \cdot\left[x_{f 1, k}+(i-1) \cdot s_{f}\right]
$$

The second column includes the length of the major semi-axis of the semi-ellipse, $a$. For a generic i-th strip, $a_{i, k}$ can be determined from:

$a_{i, k}=\frac{L_{f i, k}}{2} \cdot \sin \alpha_{f i, k}\left[\frac{1}{\sin \left(\alpha_{f i, k}+\beta+\theta\right)}+\frac{1}{\sin \left(\theta+\beta-\alpha_{f i, k}\right)}\right]$

The third column stores the values of the position, along the $O X$ axis, of the center of the i-th ellipse $X_{o}$. For a generic i-th semi-ellipse, $X_{o i, k}$ can be calculated from: $e_{2 P i, k}=L_{f i, k} \cdot \tan \alpha_{f i, k}$

The sixth column includes the values of the length of the minor semi-axis of the semi-ellipse, $b$. For a generic i-th semi-ellipse $b_{i, k}$ can be calculated from:

$b_{i, k}=\sqrt{\frac{a_{i, k}^{2} \cdot e_{2 P i, k}^{2}}{\left(a_{i, k}^{2}-e_{1 P i, k}^{2}\right)}}$

The seventh column includes the values of the position, along the $O X$ axis, of the leftward vertex of the semi-ellipse along its major axis, $v_{1}$. For a generic i-th semi-ellipse $v_{1 i, k}$ can be calculated from:

$v_{1 i, k}=X_{o i, k}-a_{i, k}$

The eight column includes the values of the position, along the $O X$ axis, of the rightward vertex of the

$$
\text { for }\left(x_{f i, k}<\frac{h_{w}}{2} \cdot(\cot \theta+\cot \beta)\right)
$$

$$
\left\{X_{o i, k}=\frac{h_{w}}{\sin \theta}-\frac{\sin \left(\beta-\alpha_{f i, k}\right)}{\sin \left(\beta+\theta-\alpha_{f i, k}\right)} \cdot\left[h_{w} \cdot(\cot \theta+\cot \beta)-x_{f i, k}\right]-a_{i, k} \text { for }\left(x_{f i, k} \geq \frac{h_{w}}{2} \cdot(\cot \theta+\cot \beta)\right)\right.
$$

semi-ellipse along its major axis, $v_{2}$. For a generic i-th semiellipse $v_{2 i, k}$ can be calculated from:

$$
v_{2 i, k}=X_{o i, k}+a_{i, k}
$$

\section{Determination of the Coefficients of the Semi-Ellipses}

The equation of a generic $\mathrm{i}$-th semi-ellipse of the $\mathrm{k}$-th configuration, in the crack plane reference system has to be determined i.e.:

$$
Y_{i, k}(X)=+\sqrt{-\frac{\left(E_{i 1, k} \cdot X^{2}+E_{i 3, k} \cdot X+E_{i 4, k}\right)}{E_{i 2, k}}}
$$
relevant ellipse, $e_{2 P i}$. For a generic $\mathrm{i}$-th ellipse of the k-th configuration, $e_{2 P i, k}$ can be calculated from: 
For this purpose, the coefficients of the semi-ellipses are stored in the $\underline{E}$ matrix that, for the k-th configuration $\left(\underline{E}_{k}\right)$ has $N_{f, k} \times 4$ dimensions. The first to fourth columns of the $\underline{E}$ matrix store the values of the coefficients of the semiellipses. For a generic i-th semi-ellipse of the k-th configuration, these coefficients can be calculated from: of the assumed crack origin. The general term $X_{i j, k}^{p 1 / 2}$ (represents both $X_{i j, k}^{p 1}$ and $X_{i j, k}^{p 2}$ ) calculated as above specified, will be stored in the $j$-th column of the $i$-th row of the relevant auxiliary matrix $\underline{X}_{k}^{p 1 / 2}$ if it is such as to satisfy the following condition:

$$
E_{i 1, k}=b_{i, k}^{2} ; E_{i 2, k}=a_{i, k}^{2} ; E_{i 3, k}=-2 \cdot b_{i, k}^{2} \cdot X_{o i, k} ; E_{i 4, k}=b_{i, k}^{2} \cdot X_{o i, k}^{2}-a_{i, k}^{2} \cdot b_{i, k}^{2}
$$

\section{Determination of the Auxiliary Matrices of Integration Points}

It is worth determining, even if they are not strictly necessary for the implementation of the algorithm, some auxiliary matrices i.e. $\underline{X}_{k}^{p 1}, \underline{X}_{k}^{p 2}, \underline{X}_{k}^{q}, \underline{Y}_{k}^{e}, \underline{M}_{k}, \underline{N}_{k}, \underline{Q}_{k}$ since they condense some operations that, otherwise, should be repeated several times. $\underline{X}^{p 1}$ and $\underline{X}^{p 2}$ are two $N_{f} \times N_{f}$ dimensions symmetric matrices containing, respectively, the abscissa of the first, $X_{i j}^{p 1}$, and second, $X_{i j}^{p 2}$, intersection points, if actually existing, between the $\mathrm{i}$-th and $\mathrm{j}$-th semiellipses. For the k-th configuration, the generic terms $X_{i j, k}^{p 1}$ and $X_{i j, k}^{p 2}$ of the $\underline{X}_{k}^{p 1}$ and $\underline{X}_{k}^{p 2}$ matrices are determined, respectively, from Eq. (27.1) and (27.2) if the following conditions, Eqs. (26.1-2), are satisfied:

$$
\left(E_{j 1, k} \cdot E_{i 2, k}-E_{i 1, k} \cdot E_{j 2, k}\right) \neq 0
$$

$\Delta_{i j, k}=\left[E_{i 2, k} \cdot E_{j 3, k}-E_{i 3, k} \cdot E_{j 2, k}\right]^{2}-4 \cdot\left[E_{j 1, k} \cdot E_{i 2, k}-E_{i 1, k} \cdot E_{j 2, k}\right] \cdot\left[E_{i 2, k} \cdot E_{j 3, k}-E_{j 2, k} \cdot E_{i 3, k}\right]>0$

$$
X_{i j, k}^{p 1}=\frac{-\left(E_{i 2, k} \cdot E_{j 3, k}-E_{j 2, k} \cdot E_{i 3, k}\right)-\sqrt{\Delta_{i j, k}}}{2 \cdot\left(E_{j 1, k} \cdot E_{i 2, k}-E_{i 1, k} \cdot E_{j 2, k}\right)}
$$$$
X_{i j, k}^{p 2}=\frac{-\left(E_{i 2, k} \cdot E_{j 3, k}-E_{j 2, k} \cdot E_{i 3, k}\right)+\sqrt{\Delta_{i j, k}}}{2\left(E_{j 1, k} \cdot E_{i 2, k}-E_{i 1, k} \cdot E_{j 2, k}\right)}
$$

Otherwise, if the following condition is satisfied:

$$
\left(E_{j 1, k} \cdot E_{i 2, k}-E_{i 1, k} \cdot E_{j 2, k}\right)=0
$$

the $i$-th and $j$-th semi-ellipses are intersecting in only one point, and the abscissa in the $\mathrm{OX}$ axis is given by:

$$
X_{i j, k}^{p 1}=-\frac{\left(E_{i 2, k} \cdot E_{j 4, k}-E_{j 2, k} \cdot E_{i 4, k}\right)}{\left(E_{i 2, k} \cdot E_{j 3, k}-E_{j 2, k} \cdot E_{i 3, k}\right)}
$$

In this case a "non-value", represented by an asterisk, is assigned to the corresponding cell of the $\underline{X}_{k}^{p 2}$ matrix, i.e.:

$X_{i j, k}^{p 2}=*$

Note that a "non-value" term is not zero since this latter has a physical meaning representing the position, in $O X Z$,

$$
-\frac{\left[E_{i 1, k} \cdot\left(X_{i j, k}^{p 1 / 2}\right)^{2}+E_{i 3, k} \cdot\left(X_{i j, k}^{p 1 / 2}\right)+E_{i 4, k}\right]}{E_{i 2, k}}>0
$$

If for the general solution $X_{i j, k}^{p 1 / 2}$, neither the conditions of Eqs. (26 and 31) nor Eqs. (28 and31) are satisfied, the corresponding cell of the relevant matrix $\underline{X}_{k}^{p 1 / 2}$ has to be filled with a "non value", e.g., an asterisk. Throughout the following calculations, each time neither the existence nor acceptance conditions of a real value are fulfilled, the corresponding cell has to be filled with a "non-value". $\underline{X}^{q}$ is a $N_{f} \times 2$ dimensions matrix containing, in each i-th row, the abscissa of the left $X_{i 1}^{q}$ and right $X_{i 2}^{q}$ intersection, if actually existing, of the relevant $\mathrm{i}$-th semi-ellipse with the straight line $Y=b_{w} / 2$. For the general k-th configuration, the first column term of the i-th row, $X_{i 1, k}^{q}$, and the second

column one, $X_{i 2, k}^{q}$, of the $\underline{X}_{k}^{q}$ matrix are calculated, respectively, from the following Eqs.:

$$
X_{i 1, k}^{q}=\frac{-E_{i 3, k}-\sqrt{E_{i 3, k}^{2}-4 E_{i 1, k} \cdot\left(E_{i 2, k} \cdot b_{w}^{2} / 4+E_{i 4, k}\right)}}{2 \cdot E_{i 1, k}}
$$

$$
X_{i 2, k}^{q}=\frac{-E_{i 3, k}+\sqrt{E_{i 3, k}^{2}-4 \cdot E_{i 1, k}\left(E_{i 2, k} \cdot b_{w}^{2} / 4+E_{i 4, k}\right)}}{2 \cdot E_{i 1, k}}
$$

if the following condition is satisfied:

$$
\Delta_{i, k}=E_{i 3, k}^{2}-4 \cdot E_{i 1, k}\left(E_{i 2, k} \cdot b_{w}^{2} / 4+E_{i 4, k}\right) \geq 0
$$

$\underline{Y}^{e}$ is a $N_{f} \times 2$ dimensions matrix containing, in each ith row, the ordinate assumed by the i-th semi-ellipse in correspondence of $X=0$, and in correspondence of $X=L_{d}$, if the semi-ellipse actually passes through those abscissa values. For the generic k-th configuration, the first term $Y_{i 1, k}^{e}$ of the i-th row of the $\underline{Y}_{k}^{e}$ matrix is a real number, indicating 
that the relevant semi-ellipse effectively passes through $X=0$ if the following condition is satisfied:

$-\frac{E_{i 4, k}}{E_{i 2, k}} \geq 0$

and in that case the corresponding value $Y_{i 1, k}^{e}$ is equal to:

$Y_{i 1, k}^{e}=+\sqrt{-\frac{E_{i 4, k}}{E_{i 2, k}}}$

Likewise, the second term $Y_{i 2, k}^{e}$ of the i-th row of the matrix $\underline{Y}_{k}^{e}$ is constituted of a real value, meaning that the relevant $\mathrm{i}$-th semi-ellipse of the k-th configuration effectively passes through $X=L_{d}$ if the following condition is satisfied:

$$
-\frac{\left(E_{i 4, k}+E_{i 3, k} \cdot L_{d}+E_{i 1, k} \cdot L_{d}^{2}\right)}{E_{i 2, k}} \geq 0
$$

and the corresponding value $Y_{i 2, k}^{e}$ is determined by the following expression:

$Y_{i 2, k}^{e}=+\sqrt{-\frac{\left(E_{i 1, k} \cdot L_{d}^{2}+E_{i 3, k} \cdot L_{d}+E_{i 4, k}\right)}{E_{i 2, k}}}$

$\underline{M}, \underline{N}, \underline{Q}$ are $N_{f} \times N_{f}$ dimensions matrices containing, respectively, the coefficients $M_{i j}, N_{i j}$ and $Q_{i j}$ with $i, j=1, \ldots ., N_{f}$. For the generic k-th configuration, the general terms $M_{i j, k}, N_{i j, k}, Q_{i j, k}$ of the $\underline{M}_{k}, \underline{N}_{k}$ and $\underline{Q}_{k}$ matrices are calculated as follows:

$M_{i j, k}=\left[\left(\frac{E_{i 1, k}}{E_{i 2, k}}-\frac{E_{j 1, k}}{E_{j 2, k}}\right)\right] ; N_{i j, k}=\left[\left(\frac{E_{i 3, k}}{E_{i 2, k}}-\frac{E_{j 3, k}}{E_{j 2, k}}\right)\right] ;$

$Q_{i j, k}=\left[\left(\frac{E_{i 4, k}}{E_{i 2, k}}-\frac{E_{j 4, k}}{E_{j 2, k}}\right)\right]$

where $E_{i 1, k}, E_{i 2, k}, E_{i 3, k}, E_{i 4, k}$ and $E_{j 1, k}, E_{j 2, k}, E_{j 3, k}$, $E_{j 4, k}$ are, respectively, the coefficients of the i-th and $\mathrm{j}$-th semi-ellipses in the k-th configuration stored in the relevant rows of the $\underline{E}_{k}$ matrix.

Determination of the Integration Points in the Non Linear Range $\underline{X}_{k}^{n l i n}$

$\underline{X}^{n l i n}$ is a $N_{f} \times n^{\text {nlin }}$ dimensions matrix containing, in the $i$-th row, the couples of abscissa values constituting limits of the integration intervals for the relevant $\mathrm{i}$-th semiellipse equation $Y_{i}(X)$. For the k-th configuration, the matrix $\underline{X}_{k}^{n l i n}$ has $N_{f, k} \times n_{k}^{n l i n}$ dimensions where $n_{k}^{\text {nlin }}$ is the maximum number of real values of integration limits amongst all the $N_{f, k}$ ellipses of that configuration (an even number). To evaluate $\underline{X}_{k}^{\text {nlin }}$, five other auxiliary matrices $\underline{X}_{k}^{n l i n 1}, \underline{X}_{k}^{n l i n 2}, \underline{X}_{k}^{n l i n 3}, \underline{X}_{k}^{n l i n 4}, \underline{X}_{k}^{n l i n 5}$ have to be determined, based on both the auxiliary ones $\underline{X}_{k}^{p 1}, \underline{X}_{k}^{p 2}, \underline{X}_{k}^{q}$, $\underline{Y}_{k}^{e}, \underline{M}_{k}, \underline{N}_{k}, \underline{Q}_{k}$, output of the previous block of calculations, and the matrix of the semi-ellipses geometrical properties, $\underline{G}_{k}$.

$\underline{X}^{\text {nlin1 }}$ and $\underline{X}^{\text {nlin2 }}$ are two $N_{f} \times N_{f}$ dimensions matrices containing, in the i-th row, the abscissa values, amongst those already calculated and stored in the corresponding $\mathrm{i}$-th row, respectively, of the auxiliary matrices $\underline{X}^{p 1}$ and $\underline{X}^{p 2}$, that, according to the acceptance conditions hereafter specified, effectively constitute useful integration limits for the relevant i-th semi-ellipse equation. For the k-th configuration, the general $\mathrm{j}$-th term $X_{i j, k}^{n l i n 1 / 2}$ of the i-th row of the $\underline{X}_{k}^{n l i n 1 / 2}$ matrix is set equal to the corresponding term $X_{i j, k}^{p 1 / 2}$ of the corresponding auxiliary matrix $\underline{X}_{k}^{p 1 / 2}$, i.e.:

$X_{i j, k}^{n l i n 1 / 2}=X_{i j, k}^{p 1 / 2}$

if $X_{i j, k}^{p 1 / 2}$ is such as to satisfy, for the $\mathrm{i}$-th semi-ellipse, the following acceptance conditions:

$$
\begin{aligned}
& 0<X_{i j, k}^{p 1 / 2}<L_{d} \text { and } Y_{i, k}\left(X_{i j, k}^{p 1 / 2}\right)<\frac{b_{w}}{2} \\
& M_{i h, k} \cdot\left(X_{i j, k}^{p 1 / 2}\right)^{2}+N_{i h, k} \cdot\left(X_{i j, k}^{p 1 / 2}\right)+Q_{i h, k} \leq 0 \\
& \text { for } h=1 \ldots . N_{f, k} \\
& \iint 0<\left(X_{i j, k}^{p 1 / 2}+\Delta X\right)<L_{d} \text { and } \\
& \left\{0<Y_{i, k}\left(X_{i j, k}^{p 1 / 2}+\Delta X\right)<\frac{b_{w}}{2}\right. \text { and } \\
& M_{i h, k} \cdot\left(X_{i j, k}^{p 1 / 2}+\Delta X\right)^{2}+N_{i h, k} \cdot\left(X_{i j, k}^{p 1 / 2}+\Delta X\right)+Q_{i h, k}<0 \\
& \forall h=1 \ldots . N_{f, k} \text { and } h \neq i \\
& \text { or } \\
& 0<\left(X_{i j, k}^{p 1 / 2}-\Delta X\right)<L_{d} \text { and } \\
& 0<Y_{i, k}\left(X_{i j, k}^{p 1 / 2}-\Delta X\right)<\frac{b_{w}}{2} \text { and } \\
& M_{i h, k} \cdot\left(X_{i j, k}^{p 1 / 2}-\Delta X\right)^{2}+N_{i h, k} \cdot\left(X_{i j, k}^{p 1 / 2}-\Delta X\right)+Q_{i h, k}<0 \\
& \forall h=1 \ldots . N_{f, k} \text { and } h \neq i \\
& X_{i j, k}^{p 1 / 2} \neq X_{i g, k}^{n l i n 1 / 2} \quad \text { with } g=1, \ldots . .,(j-1)
\end{aligned}
$$

in which the term $\Delta X$ indicates an infinitesimally small length along the $O X$ axis. If at least one of the above condi- 
tions is not fulfilled by the auxiliary value $X_{i j, k}^{p 1 / 2}$, the corresponding effective term $X_{i j, k}^{n l i n 1 / 2}$ has to be set equal to "nonvalue". $\underline{X}^{n l i n 3}$ is a $N_{f} \times 2$ matrix containing, in the first and second column of the i-th row, $X_{i 1}^{\text {nlin3 } 3}$ and $X_{i 2}^{\text {nlin3 } 3}$, the abscissa values of the left and right intersection points of the relevant semi-ellipse with the straight line $Y=b_{w} / 2$ that result effective for the integration of the corresponding equation $Y_{i}(X)$. For the k-th configuration, the term $X_{i 1, k}^{\text {nlin3 }}$ of the i-th row of the $\underline{X}_{k}^{\text {nlin3 } 3}$ matrix is set equal to the corresponding term $X_{i 1, k}^{q}$, i.e.:

$X_{i 1, k}^{n \operatorname{lin} 3}=X_{i 1, k}^{q}$

if $X_{i 1, k}^{q}$ is such as to satisfy the following acceptance conditions:

values result to be effective integration limits for the relevant semi-ellipse $Y_{i}(X)$. For the generic k-th configuration, the first column term of the i-th row, $X_{i 1, k}^{n l i n 4}$, of the $\underline{X}_{k}^{n l i n 4}$ matrix has to be set equal to zero, i.e.:

$$
X_{i 1, k}^{n \operatorname{lin} 4}=0
$$

if the ordinate value, $Y_{i 1, k}^{e}$, contained in the corresponding cell of the $\underline{Y}_{k}^{e}$ matrix satisfies the following conditions:

$$
\left\{\begin{array}{l}
0<Y_{i 1, k}^{e}<\frac{b_{w}}{2} \\
Q_{i j, k} \leq 0 \quad \forall j=1, \ldots \ldots, N_{f, k} \\
0<Y_{i, k}(\Delta X)<\frac{b_{w}}{2} \\
M_{i j, k} \cdot(\Delta X)^{2}+N_{i j, k} \cdot(\Delta X)+Q_{i j, k}<0 \quad \forall j=1, \ldots ., N_{f, k} j \neq i
\end{array}\right.
$$

$$
\left\{\begin{array}{l}
0 \leq X_{i 1, k}^{q} \leq L_{d} \\
M_{i j, k} \cdot\left(X_{i 1, k}^{q}\right)^{2}+N_{i j, k} \cdot\left(X_{i 1, k}^{q}\right)+Q_{i j, k} \leq 0 \quad \text { for } \forall j=1, \ldots \ldots, N_{f, k} \\
0<\left(X_{i 1, k}^{q}-\Delta X\right)<L_{d} \\
Y_{i, k}\left(X_{i 1, k}^{q}-\Delta X\right)<\frac{b_{w}}{2} \\
M_{i j, k} \cdot\left(X_{i 1, k}^{q}-\Delta X\right)^{2}+N_{i j, k} \cdot\left(X_{i 1, k}^{q}-\Delta X\right)+Q_{i j, k}<0 \quad \forall j=1, \ldots . ., N_{f, k} \quad j \neq i
\end{array}\right.
$$

Likewise, the second column term of the i-th row, $X_{i 2, k}^{n \operatorname{lin} 4}$, of the $\underline{X}_{k}^{n l i n 4}$ matrix has to be set equal to $L_{d}$, i.e.: ing auxiliary term $X_{i 2, k}^{q}$, i.e.:

$X_{i 2, k}^{n l i n 3}=X_{i 2, k}^{q}$

$$
X_{i 2, k}^{n \operatorname{lin} 4}=L_{d}
$$

if $X_{i 2, k}^{q}$ meets the following acceptance condition:

$$
\left\{\begin{array}{l}
0 \leq X_{i 2, k}^{q} \leq L_{d} \\
M_{i j, k} \cdot\left(X_{i 2, k}^{q}\right)^{2}+N_{i j, k} \cdot\left(X_{i 2, k}^{q}\right)+Q_{i j, k} \leq 0 \quad \forall j=1, \ldots ., N_{f, k} \\
0<\left(X_{i 2, k}^{q}+\Delta X\right)<L_{d} \\
Y_{i, k}\left(X_{i 2, k}^{q}+\Delta X\right)<\frac{b_{w}}{2} \\
M_{i j, k} \cdot\left(X_{i 2, k}^{q}+\Delta X\right)^{2}+N_{i j, k} \cdot\left(X_{i 2, k}^{q}+\Delta X\right)+Q_{i j, k}<0 \quad \forall j=1, \ldots, N_{f, k} \quad j \neq i
\end{array}\right.
$$

$\underline{X}^{n l i n 4}$ is a $N_{f} \times 2$ dimensions matrix containing, in the first cell of the i-th row, the null abscissa value, $X_{i 1}^{n l i n} 4=0$, and the $L_{d}$ value in the second cell, $X_{i 2}^{n \operatorname{lin} 4}=L_{d}$, if those if the ordinate value, $Y_{i 2, k}^{e}$, contained in the corresponding cell of the $\underline{Y}_{k}^{e}$ matrix satisfies the following conditions: 


$$
\left\{\begin{array}{l}
0<Y_{i 2, k}^{e}<\frac{b_{w}}{2} \\
M_{i j, k} \cdot L_{d}^{2}+N_{i j, k} \cdot L_{d}+Q_{i j, k} \leq 0 \quad \forall j=1, \ldots ., N_{f, k} \\
0<Y_{i, k}\left(L_{d}-\Delta X\right)<\frac{b_{w}}{2} \\
M_{i j, k} \cdot\left(L_{d}-\Delta X\right)^{2}+N_{i j, k} \cdot\left(L_{d}-\Delta X\right)+Q_{i j, k}<0 \quad \forall j=1, \ldots . ., N_{f, k} \quad j \neq i
\end{array}\right.
$$

$\underline{X}^{n l i n 5}$ is a $N_{f} \times 2$ dimensions matrix containing the abscissa of the vertices of the major semi-axis of the semi-ellipse that constitute effective integration extremities for the ellipses.

For the k-th configuration, the first column term of the ith row, $X_{i 1, k}^{n l i n 5}$, of the $\underline{X}_{k}^{n l i n 5}$ matrix has to be set equal to the term $G_{i 7, k}$, stored in the seventh column cell of the corresponding i-th row of the matrix $\underline{G}_{k}$ i.e.:

$X_{i 1, k}^{n l i n 5}=G_{i 7, k}$

if $G_{i 7, k}$ satisfies the following conditions: tion (the integrand function is nonlinear in the $\mathrm{X}$ variable). For the k-th configuration, the general i-th term, $n_{i, k}^{n l i n}$, of the $\underline{n}_{k}^{\text {nlin }}$ vector is equal to the number of real values present amongst all the terms stored in the corresponding i-th row of all the auxiliary matrices, i.e.:

$n_{i, k}^{\text {nlin }}=$ real numbers $\left\{X_{i, k}^{n \operatorname{lin} 1} ; X_{i, k}^{n \operatorname{lin} 2} ; X_{i, k}^{n l i n 3} ; X_{i, k}^{n l i n 4} ; X_{i, k}^{n l i n 5}\right\}$

The number of columns of the $\underline{X}_{k}^{\text {nlin }}$ matrix, $n_{k}^{\text {nlin }}$, is equal to the maximum number of effective values among all the semi-ellipses for the $\mathrm{k}$-th configuration, i.e.:

$$
\left\{\begin{array}{l}
0 \leq G_{i 7, k} \leq L_{d} \\
-\frac{\left[E_{j 1, k} \cdot\left(G_{i 7, k}\right)^{2}+E_{j 3, k} \cdot\left(G_{i 7, k}\right)+E_{j 4, k}\right]}{E_{j 2, k}} \leq 0 \quad \forall j=1 ; \ldots . . ; N_{f, k} \\
0<\left(G_{i 7, k}+\Delta X\right)<L_{d} \\
M_{i j, k} \cdot\left(G_{i 7, k}+\Delta X\right)^{2}+N_{i j, k} \cdot\left(G_{i 7, k}+\Delta X\right)+Q_{i j, k}<0 \quad \forall j=1 ; \ldots . ; N_{f, k} \text { and } j \neq i
\end{array}\right.
$$

Likewise, the second column term of the i-th row, $X_{i 2, k}^{n l i n 5}$, has to be set equal to the term $G_{i 8, k}$, stored in the 8 -th column cell of the $\mathrm{i}$-th row of the previously determined $\underline{G}_{k}$ matrix i.e.:

$X_{i 2, k}^{n l i n 5}=G_{i 8, k}$

if $G_{i 8, k}$ satisfies the following conditions: $n_{k}^{\text {nlin }}=\max \left\{n_{i, k}^{\text {nlin }}\right\}$ with $i=1 ; \ldots ; N_{f, k}$

The $\underline{X}_{k}^{n l i n}$ matrix is then built by joining, for each i-th row corresponding to the $\mathrm{i}$-th semi-ellipse, the effective terms, discarding the "non-values", present in the corresponding i-th row of the auxiliary matrices $\underline{X}_{k}^{n \operatorname{lin} 1}, \underline{X}_{k}^{n \operatorname{lin} 2}$, $\underline{X}_{k}^{n l i n 3}, \underline{X}_{k}^{n l i n 4}, \underline{X}_{k}^{n l i n 5}$ and sorting them in increasing order.

$$
\left\{\begin{array}{l}
0 \leq G_{i 8, k} \leq L_{d} \\
-\frac{\left[E_{j 1, k} \cdot\left(G_{i 8, k}\right)^{2}+E_{j 3, k} \cdot\left(G_{i 8, k}\right)+E_{j 4, k}\right]}{E_{j 2, k}} \leq 0 \quad \forall j=1 ; \ldots . . ; N_{f, k} \\
0<\left(G_{i 8, k}-\Delta X\right)<L_{d} \\
M_{i j, k} \cdot\left(G_{i 8, k}-\Delta X\right)^{2}+N_{i j, k} \cdot\left(G_{i 8, k}-\Delta X\right)+Q_{i j, k}<0 \quad \forall j=1 ; \ldots . ; N_{f, k} \text { and } j \neq i
\end{array}\right.
$$

$\underline{n}^{\text {nlin }}$ is a $N_{f} \times 1$ vector containing, in the $\mathrm{i}$-th row, the maximum number of real abscissa values constituting effective integration limits for the relevant $\mathrm{i}$-th semi-ellipse equa-
For instance, the transpose $\left(\underline{X}_{k}^{\text {nlin }}\right)^{T}$ of the final $\underline{X}_{k}^{n l i n}$ matrix for the example of Fig. 11 is as follows (see also Fig. 14): 


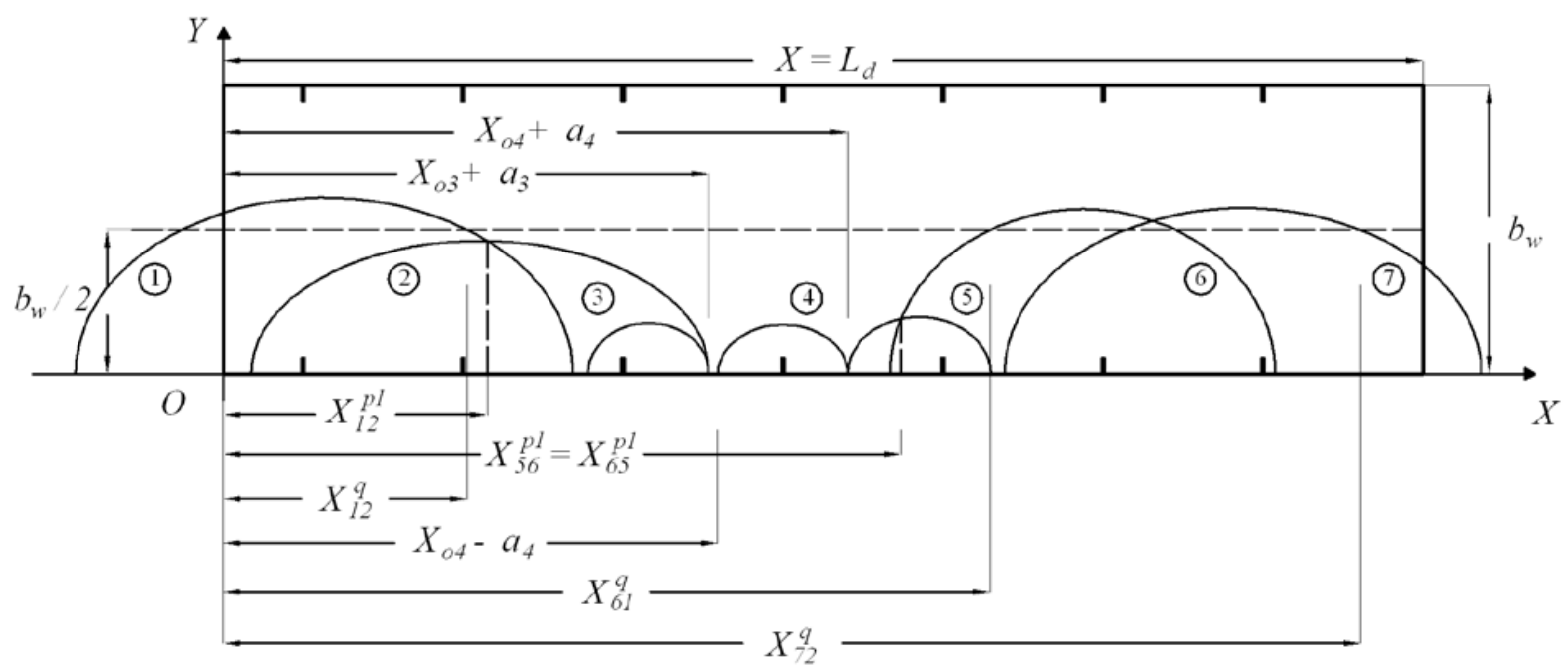

Fig. (14). Determination of the effective matrix of the integration points in the non-linear range $\underline{X}_{k}^{\prime \prime \prime \prime \prime \prime}$.

$$
\left(\underline{X}_{k}^{n l i n}\right)^{T}=\left[\begin{array}{ccccccc}
X_{12}^{q} & X_{21}^{p 1} & * & \left(X_{o 4}-a_{4}\right) & \left(X_{o 5}-a_{5}\right) & X_{65}^{p 1} & X_{72}^{q} \\
X_{12}^{p 1} & \left(X_{o 2}+a_{2}\right) & * & \left(X_{o 4}+a_{4}\right) & X_{56}^{p 1} & X_{61}^{q} & L_{d}
\end{array}\right]
$$

\section{Determination of the Integration Points in the Linear} Range $\underline{X}_{k}^{\text {lin }}$

$\underline{X}^{\text {lin }}$ is a $N_{f} \times n^{\text {lin }}$ dimensions matrix containing, in the i-th row, the couples of abscissa values constituting limits of the integration intervals, in correspondence of the i-th semiellipse, of the equation $Y=b_{w} / 2$. For the generic k-th configuration, the matrix $\underline{X}_{k}^{\text {lin }}$ has $N_{f, k} \times n_{k}^{\text {lin }}$ dimensions where $n_{k}^{\text {lin }}$ is the maximum number of real values of integration limits amongst all the $N_{f, k}$ semi-ellipses of that configuration (an even number). To evaluate $\underline{X}_{k}^{\text {lin }}$, four other auxiliary matrices $\underline{X}_{k}^{\operatorname{lin} 1}, \underline{X}_{k}^{\operatorname{lin} 2}, \underline{X}_{k}^{\operatorname{lin} 3}, \underline{X}_{k}^{\operatorname{lin} 4}$ have to be determined, based on the auxiliary ones $\underline{X}_{k}^{p 1}, \underline{X}_{k}^{p 2}, \underline{X}_{k}^{q}, \underline{Y}_{k}^{e}$, $\underline{M}_{k}, \underline{N}_{k}, \underline{Q}_{k}$, already built. $\underline{X}^{\operatorname{lin} 1}$ and $\underline{X}^{\operatorname{lin} 2}$ are two $N_{f} \times N_{f}$ dimensions matrices containing, in the i-th row, the abscissa values, amongst those already calculated and stored in the corresponding $\mathrm{i}$-th row of the auxiliary matrices $\underline{X}^{p 1}$ and $\underline{X}^{p 2}$, respectively, that, according to the acceptance conditions hereafter specified, effectively constitute useful integration limits for the linear range ascribed to the relevant i-th semi-ellipse. For the k-th configuration, the general $\mathrm{j}$-th term $X_{i j, k}^{l i n 1 / 2}$ of the $\mathrm{i}$-th row of the $\underline{X}_{k}^{l i n 1 / 2}$ matrix is set equal to the corresponding term $X_{i j, k}^{p 1 / 2}$ of the corresponding auxiliary matrix $\underline{X}_{k}^{p 1 / 2}$, i.e.:

$X_{i j, k}^{l i n 1 / 2}=X_{i j, k}^{p 1 / 2}$ if $X_{i j, k}^{p 1 / 2}$ is such as to satisfy, for the $\mathrm{i}$-th semi-ellipse, the following acceptance conditions:

$$
\begin{aligned}
& \left\{\begin{array}{l}
0<X_{i j, k}^{p 1 / 2}<L_{d} \text { and } Y_{i, k}\left(X_{i j, k}^{p 1 / 2}\right)>\frac{b_{w}}{2} \\
M_{i h, k} \cdot\left(X_{i j, k}^{p 1 / 2}\right)^{2}+N_{i h, k} \cdot\left(X_{i j, k}^{p 1 / 2}\right)+Q_{i h, k} \leq 0 \text { for } h=1 \ldots N_{f, k} \\
\left\{\begin{array}{l}
0<\left(X_{i j, k}^{p 1 / 2}+\Delta X\right)<L_{d} \\
Y_{i, k}\left(X_{i j, k}^{p 1 / 2}+\Delta X\right)>\frac{b_{w}}{2} \\
M_{i h, k} \cdot\left(X_{i j, k}^{p 1 / 2}+\Delta X\right)^{2}+N_{i h, k} \cdot\left(X_{i j, k}^{p 1 / 2}+\Delta X\right)+Q_{i h, k}<0 \quad \forall h \neq i
\end{array}\right.
\end{array}\right. \\
& \text { or } \\
& \int 0<\left(X_{i j, k}^{p 1 / 2}-\Delta X\right)<L_{d} \text { and } \\
& Y_{i, k}\left(X_{i j, k}^{p 1 / 2}-\Delta X\right)>\frac{b_{w}}{2} \text { and } \\
& M_{i h, k} \cdot\left(X_{i j, k}^{p 1 / 2}-\Delta X\right)^{2}+N_{i h, k} \cdot\left(X_{i j, k}^{p 1 / 2}-\Delta X\right)+Q_{i h, k}<0 \forall h \neq i \\
& X_{i j, k}^{p 1 / 2} \neq X_{i g, k}^{l i n 1 / 2} \text { with } g=1, \ldots \ldots,(j-1)
\end{aligned}
$$

Note that $X_{i j, k}^{p 1 / 2}$ in Eq. (57) represents the two possible solutions, $X_{i j, k}^{p 1}$ and $X_{i j, k}^{p 2} \cdot \underline{X}^{l i n 3}$ is a $N_{f} \times 2$ matrix containing, in the first and second columns of the i-th row, $X_{i 1}^{\operatorname{lin} 3}$ and $X_{i 2}^{\operatorname{lin} 3}$, respectively, the abscissa values of the left and right intersection points of the relevant semi-ellipse with the straight line $Y=b_{w} / 2$ that result effective for the integration of the corresponding equation $Y=b_{w} / 2$. For the k-th configuration, the first column term of the i-th row, $X_{i 1, k}^{\operatorname{lin} 3}$, of 


$$
\left\{\begin{array}{l}
0 \leq X_{i 1, k}^{q} \leq L_{d} \\
M_{i j, k} \cdot\left(X_{i 1, k}^{q}\right)^{2}+N_{i j, k} \cdot\left(X_{i 1, k}^{q}\right)+Q_{i j, k} \leq 0 \quad \forall j=1 \ldots . N_{f, k} \\
0<\left(X_{i 1, k}^{q}+\Delta X\right)<L_{d} \\
Y_{i, k}\left(X_{i 1, k}^{q}+\Delta X\right)>\frac{b_{w}}{2} \\
M_{i j, k} \cdot\left(X_{i 1, k}^{q}+\Delta X\right)^{2}+N_{i j, k} \cdot\left(X_{i 1, k}^{q}+\Delta X\right)+Q_{i j, k}<0 \quad \forall \quad j=1 . . N_{f, k} \quad j \neq i
\end{array}\right.
$$

the $\underline{X}_{k}^{\text {lin3 } 3}$ matrix is set equal to the corresponding term $X_{i 1, k}^{q}$ of the auxiliary matrix $\underline{X}_{k}^{q}$, i.e.:

$X_{i 1, k}^{\operatorname{lin} 3}=X_{i 1, k}^{q}$

if $X_{i 1, k}^{q}$ satisfies the following conditions:

Likewise, the second column term of the i-th row $X_{i 2, k}^{\operatorname{lin} 3}$ is set equal to the corresponding auxiliary term $X_{i 2, k}^{q}$, i.e.:

$X_{i 2, k}^{\operatorname{lin} 3}=X_{i 2, k}^{q}$

if $X_{i 2, k}^{q}$ meets the following acceptance condition:

$$
\left\{\begin{array}{l}
Y_{i 1, k}^{e}>\frac{b_{w}}{2} \\
Q_{i j, k} \leq 0 \quad \forall j=1 \ldots . N_{f, k} \\
Y_{i, k}(\Delta X)>\frac{b_{w}}{2} \\
M_{i j, k} \cdot(\Delta X)^{2}+N_{i j, k} \cdot(\Delta X)+Q_{i j, k}<0 \forall j=1 \ldots N_{f, k} j \neq i
\end{array}\right.
$$

Likewise, the second column term of the i-th row, $X_{i 2, k}^{\text {lin } 4}$, of the $\underline{X}_{k}^{\text {lin } 4}$ matrix has to be set equal to $L_{d}$, i.e.:

$X_{i 2, k}^{\operatorname{lin} 4}=L_{d}$

$$
\left\{\begin{array}{l}
0 \leq X_{i 2, k}^{q} \leq L_{d} \\
M_{i j, k} \cdot\left(X_{i 2, k}^{q}\right)^{2}+N_{i j, k} \cdot\left(X_{i 2, k}^{q}\right)+Q_{i j, k} \leq 0 \quad \forall j=1 \ldots N_{f, k} \\
0<\left(X_{i 2, k}^{q}-\Delta X\right)<L_{d} \\
Y_{i, k}\left(X_{i 2, k}^{q}-\Delta X\right)>\frac{b_{w}}{2} \\
M_{i j, k} \cdot\left(X_{i 2, k}^{q}-\Delta X\right)^{2}+N_{i j, k} \cdot\left(X_{i 2, k}^{q}-\Delta X\right)+Q_{i j, k}<0 \quad \forall j=1 . . N_{f, k} \quad j \neq i
\end{array}\right.
$$

$\underline{X}^{\operatorname{lin} 4}$ is a $N_{f} \times 2$ dimensions matrix containing, in the first cell of the i-th row, the null abscissa value, $X_{i 1}^{\operatorname{lin} 4}=0$, and the $L_{d}$ value in the second cell, $X_{i 2}^{\operatorname{lin} 4}=L_{d}$, if those values result to be effective integration limits for the linear range ascribed to the relevant i-th semi-ellipse. For the generic k-th configuration, the first cell of the i-th row, $X_{i 1, k}^{\operatorname{lin} 4}$, of the $\underline{X}_{k}^{\text {lin4 }}$ matrix has to be set equal to zero, i.e.:

$X_{i 1, k}^{l i n 4}=0$

if the ordinate value, $Y_{i 1, k}^{e}$, contained in the corresponding cell of the $\underline{Y}_{k}^{e}$ matrix satisfies the following conditions: if the ordinate value, $Y_{i 2, k}^{e}$, contained in the corresponding cell of the matrix $\underline{Y}_{k}^{e}$ satisfies the following conditions:

$$
\begin{aligned}
& \left\{\begin{array}{l}
Y_{i 2, k}^{e}>\frac{b_{w}}{2} \\
M_{i j, k} \cdot L_{d}^{2}+N_{i j, k} \cdot L_{d}+Q_{i j, k} \leq 0 \forall j=1, \ldots, N_{f, k} \\
Y_{i, k}\left(L_{d}-\Delta X\right)>\frac{b_{w}}{2} \\
M_{i j, k} \cdot\left(L_{d}-\Delta X\right)^{2}+N_{i j, k} \cdot\left(L_{d}-\Delta X\right)+Q_{i j, k}<0 \forall j=1, \ldots ., N_{f, k} j \neq i
\end{array}\right. \\
& \underline{n}^{\text {lin }} \text { is a } N_{f} \times 1 \text { vector containing, in the i-th row, the }
\end{aligned}
$$
maximum number of real abscissa values constituting effective integration limits for the corresponding i-th semi-ellipse in the linear ranges (the integrand function is independent of the $X$ variable). For the k-th configuration, the general $\mathrm{i}$-th 


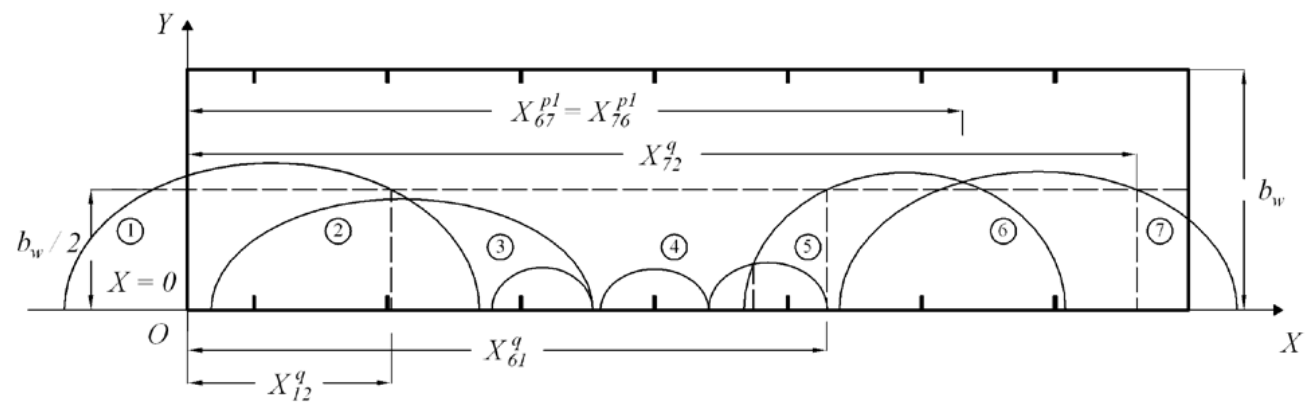

Fig. (15). Determination of the effective matrix of the integration points in the linear range.

term, $n_{i, k}^{l i n}$, of the $\underline{n}_{k}^{l i n}$ vector is equal to the number of real values present amongst all the terms stored in the corresponding $\mathrm{i}$-th row of all the auxiliary matrices, i.e.:

$n_{i, k}^{l i n}=$ real numbers $\left\{X_{i, k}^{\operatorname{lin} 1} ; X_{i, k}^{\operatorname{lin} 2} ; X_{i, k}^{\operatorname{lin} 3} ; X_{i 1, k}^{\operatorname{lin} 4}\right\}$

The number of columns of the $\underline{X}_{k}^{\text {lin }}$ matrix, $n_{k}^{\text {lin }}$, is equal to the maximum number of effective values among all the semi-ellipses for the $\mathrm{k}$-th configuration, i.e.:

$n_{k}^{\text {lin }}=\max \left\{n_{i, k}^{\text {lin }}\right\}$ with $i=1 ; \ldots ; N_{f, k}$

The $\underline{X}_{k}^{\text {lin }}$ matrix is then built by joining, for each i-th row corresponding to the $\mathrm{i}$-th semi-ellipse, the effective terms, discarding the "non-values" present in the corresponding i-th row of the auxiliary matrices $\underline{X}_{k}^{\operatorname{lin} 1}, \underline{X}_{k}^{\operatorname{lin} 2}, \underline{X}_{k}^{\operatorname{lin} 3}$, $\underline{X}_{k}^{l i n 4}$, and sorting them in increasing order. For instance, the transpose $\left(\underline{X}_{k}^{l i n}\right)^{T}$ of the final matrix $\underline{X}_{k}^{\text {lin }}$ for the example of Fig. 11 is as follows (see also Fig. 15):

$$
\left(\underline{X}_{k}^{l i n}\right)^{T}=\left[\begin{array}{ccccccc}
0 & * & * & * & * & X_{61}^{q} & X_{76}^{p 1} \\
X_{12}^{q} & * & * & * & * & X_{67}^{p 1} & X_{72}^{q}
\end{array}\right]
$$

\section{Determination of the Areas $\underline{A}_{k}$}

A is a $N_{f} \times 1$ dimension vector containing, in the i-th cell, the area ascribed to the $\mathrm{i}$-th semi-ellipse. For the $\mathrm{k}$-th configuration, the term $\mathrm{A}_{i, k}$ of the $\underline{\mathrm{A}}_{k}$ matrix is equal to:

$$
\mathrm{A}_{i, k}=\mathrm{A}_{i, k}^{n l i n}+\mathrm{A}_{i, k}^{l i n}
$$

where $\mathrm{A}_{i, k}^{\text {nlin }}$ is determined by the following equation:

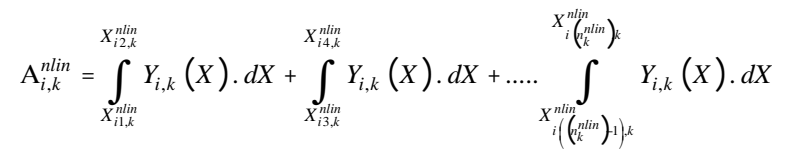

For the sake of brevity, the expression of the exact integration of the equation of the semi-ellipse is omitted but it can be found elsewhere [5]. The term $\mathrm{A}_{i, k}^{\text {lin }}$ can be obtained from:

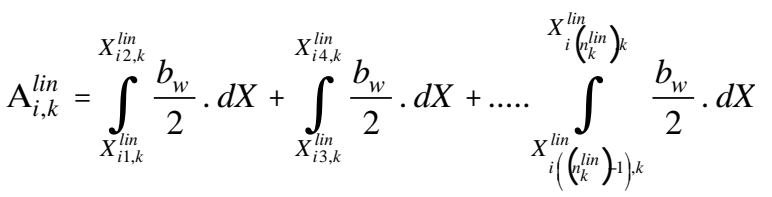

Note that in the above Eqs. (70) and (71) the abscissa values, already stored in the corresponding i-th row of $\underline{X}_{k}^{\text {nlin }}$ and $\underline{X}_{k}^{l i n}$, respectively, have to be considered integration limits by pairs in sequence.

Determination of the Shear Strength Contributions $\underline{V}_{k}^{p}$ and $V$

$\underline{V}^{p}$ is a $N_{f} \times 1$ dimension vector containing, in the $\mathrm{i}$-th cell, the shear strength contribution ascribed to the i-th strip and parallel to its orientation. For the k-th configuration, the general i-th term, $V_{f i, k}^{p}$, of the $\underline{V}_{k}^{p}$ vector is calculated by the following equation:

$V_{f i, k}^{p}=\min \left\{2 \cdot\left(a_{f}+b_{f}\right) \cdot L_{f i} \cdot \tau_{b}\left(L_{f i}\right) ;\right.$
$\left.a_{f} \cdot b_{f} \cdot f_{f u} ; \mathrm{A}_{i, k} \cdot f_{c t m} \cdot \sin (\theta+\beta)\right\}$

$\underline{V}$ is a $k \times 1$ dimension vector containing, in the k-th cell, the NSM shear strength contribution $V_{f, k}$ corresponding to the $\mathrm{k}$-th configuration. The $\mathrm{k}$-th term is equal to:

$V_{f, k}=2 \cdot \sin \beta \cdot \sum_{i=1}^{N_{f, k}} V_{f i, k}^{p}$

\section{ASSESSMENT OF THE MODEL PERFORMANCE}

The Proposed Model (PM) was used to predict the NSM contribution for the shear resistance of the beams of the experimental program. The average tensile strength of the concrete of the tested beams was estimated from the concrete average compressive strength at the age of the beam tests, and using the expressions proposed by the CEB-FIP model code 1993 [19], resulting $f_{c t m}=2.45 \mathrm{MPa}$. The results are 
Table 4. Values of $V_{f}$ Obtained from the Developed Model ( $V_{f, k}^{P M}$ ) and Experimental Recordings ( $V_{f}^{\exp }$ ) for the Experimental Program by Dias \& Barros ${ }^{12}$

\begin{tabular}{|c|c|c|c|c|c|c|c|c|c|c|}
\hline & {$[\mathrm{mm}]$} & {$\left[{ }^{\circ}\right]$} & {$\left[{ }^{\circ}\right]$} & {$[\mathrm{kN}]$} & {$[\mathrm{kN}]$} & {$[\mathrm{kN}]$} & {$[\mathrm{kN}]$} & {$[\mathrm{kN}]$} & [ ] & [ ] \\
\hline $2 \mathrm{~S} \_3 \mathrm{LV}$ & 267 & 90 & 40 & 20.88 & 13.61 & 49.28 & 13.61 & 49.28 & 1.63 & 0.45 \\
\hline 2S_5LV & 160 & 90 & 40 & 48.80 & 46.38 & 51.78 & 46.38 & 51.78 & 0.54 & 0.49 \\
\hline 2S_3LI45 & 367 & 45 & 45 & 32.62 & 22.96 & 49.83 & 22.96 & 49.83 & 1.28 & 0.59 \\
\hline 2S_5LI45 & 220 & 45 & 45 & 47.69 & 47.11 & 62.06 & 47.11 & 62.06 & 0.88 & 0.67 \\
\hline 2S_8LI45 & 138 & 45 & 36 & 83.41 & 83.16 & 88.63 & 83.16 & 88.63 & 0.48 & 0.45 \\
\hline average & & & & & & & & & 0.88 & 0.57 \\
\hline 2S_7LI60 & 139 & 60 & 37 & 72.36 & 65.35 & 74.18 & 65.35 & 74.18 & 0.84 & 0.74 \\
\hline average & & & & & & & & & 1.01 & 0.77 \\
\hline
\end{tabular}

listed in Table 4. For each beam of the experimental program, the values obtained from the developed mode (PM) are compared to the experimentally recorded shear strengthening contribution of the distinct strips' arrangements, $V_{f}^{\exp }$, with the corresponding ranges of possible analytical values. For the analysis of (Table 4), the analytical values were obtained assuming for the shear crack angle, $\theta$, the values measured in the tested beams, $\theta^{\exp }$, and also listed in Table 4. The model performance was also assessed by means of the ratios:

- $V_{f}^{\exp } / V_{f, \min }^{P M}$ of the experimental recording to the minimum value obtained by means of the PM;

- $V_{f}^{\exp } / V_{f, \text { max }}^{P M}$ of the experimental recording to the maximum value obtained by means of the PM;

The performance of the PM is absolutely satisfactory. In fact, for the series of beams with vertical strips the average of the ratios $V_{f}^{\exp } / V_{f, \text { min }}^{P M}$ and $V_{f}^{\exp } / V_{f, \max }^{P M}$ (see Table 4) are respectively 0.99 and 0.56 meaning that, on average, the recorded values fall just on the lower bound of the analytical range $\left[V_{f, \text { min }}^{P M} ; V_{f, \text { max }}^{P M}\right]$. For the series of beams with strips at $60^{\circ}$ the average value of the above two ratios are respectively 1.01 and 0.77 meaning that, on average, the experimental recordings fall in between the lower and upper bound of the analytical values. For the series of beams with strips disposed at $45^{\circ}$ the average value of the ratio $V_{f}^{\exp } / V_{f, \text { min }}^{P M}$ results to be less than unity because, the experimental value obtained in 2S_8LI45 beam was probably affected by some disturbance that did not allow the shear strengthening contri- bution of this NSM configuration to be fully mobilized. In fact, provided that, due to the interaction between subsequent strips the rate $\Delta V_{f}^{\exp } / \Delta s_{f}$ decreases by diminishing $s_{f}$, it is unrealistic that passing from $s_{f}$ of $220 \mathrm{~mm}$ (2S_5LI45 beam) to $138 \mathrm{~mm}$ (2S_8LI45 beam) the shear strength contribution decreases from 41.40 to $40.20 \mathrm{kN}$. At most, it should assume the same value of $41.40 \mathrm{kN}$.

\section{CONCLUSIONS}

The purposely intended experimental program on NSMstrengthened beams, spotlights the possibility that a failure mechanism, other than debonding, occurs, i.e. the separation of the concrete cover from the beam core. Besides, it emerges that the effectiveness of the NSM shear strengthening system may be strongly influenced by the mutual position between steel stirrups and strips. Despite the improvements introduced, the existing debonding-based model systematically provides an overestimation, the higher the smaller the spacing, of the experimentally recorded shear strengthening contribution by NSM CFRP strips. Such overestimation, as further confirmed by experimental evidence, can be ascribed to the erroneous assumption that the expected failure mechanism is debonding, regardless of the influence of both concrete tensile strength and existing stirrups/strips interaction.

A new predictive model, originated from the need for a rational explanation to the features of the above failure mechanism affecting the behavior at ultimate of RC beams shear strengthened by NSM CFRP strips, was proposed. This model assumes as possible failure mechanisms: debonding, tensile rupture of the strips and the concrete tensile fracture and allows the interaction between strips to be accounted for. 
The comparisons with the debonding-based model showed that the proposed model provided a better estimation of the experimentally recorded NSM shear strength contribution.

\section{ACKNOWLEDGEMENTS}

The authors of the present work wish to acknowledge the support provided by the "Empreiteiros Casais", S\&P®, Secil (Unibetão, Braga) and Degussa ${ }^{\circledR}$ Portugal. The study reported in this paper forms a part of the research program "SMARTREINFORCEMENT - Carbon fibre strips for the strengthening and monitoring of reinforced concrete structures" supported by ADI-IDEIA, Project n ${ }^{\circ}$ 13-05-04-FDR00031. This work has been partially carried out under the program "Dipartimento di Protezione Civile - Consorzio RELUIS", signed on 2005-07-11 (n. 540), Research Line 8, whose financial support is greatly appreciated.

\section{REFERENCES}

[1] L. De Lorenzis, and A. Nanni, "Shear Strengthening of Reinforced Concrete Beams with Near-Surface Mounted Fiber-Reinforced Polymer Rods", ACI Structural Journal, Vol. 98(1), pp. 60-68, January-February 2001.

[2] R. El-Hacha, and S. H. Rizkalla, "Near-surface-mounted fiberreinforced polymer reinforcements for flexural strengthening of concrete structures," ACI Structural Journal, Vol. 101(5) pp. 717726, 2004

[3] J. A. O. Barros, and S. J. E. Dias, "Near surface mounted CFRP laminates for shear strengthening of concrete beams", Journal of Cement and Concrete Composites, Vol. 28(3), pp. 276-292, 2006.

[4] A. Nanni, M. Di Ludovico, R. Parretti, "Shear strengthening of a PC bridge girder with NSM CFRP rectangular bars", Advances in Structural Engineering, Vol. 7(4), pp. 97-109, 2004.

[5] V. Bianco, J. A. O. Barros, G. Monti, "Shear Strengthening of RC beams by means of NSM laminates: experimental evidence and predictive models", Technical report 06-DEC/E-18, Dep. Civil Eng., School Eng. University of Minho, pp. 170, October 2006.

[6] S. J. E. Dias, J. A. O. Barros, "NSM CFRP Laminates for the Shear Strengthening of T Section RC Beams", $2^{\text {nd }}$ International fib Congress, Naples, 5-8 June 2006, Article 10-58 in CD.
American Concrete Institute, Guide for the Design and Construction of Externally Bonded FRP Systems for Strengthening Concrete Structures, ACI 440.2R-02, Farmington Hills, MI, 2002.

[8] J. M. Sena-Cruz, and J. A. O. Barros, "Bond between near-surface mounted CFRP laminate strips and concrete in structural strengthening", Journal of Composites for Construction, ASCE, Vol. 8(6), pp. 519-527, 2004.

[9] EN 206-1., "Concrete - Part 1: Specification, performance, production and conformity", European standard, CEN, pp. 69, 2000.

[10] EN 10002., "Metallic materials -Tensile testing - Part 1: Method of test (at ambient temperature)", p. 35, 1990.

[11] ISO 527-5., "Plastics - Determination of tensile properties - Part 5: Test conditions for unidirectional fiber-reinforced plastic composites", International Organization for Standardization, Genève, Switzerland, pp. 9, 1997.

[12] S. J. E. Dias, J. A. O. Barros, "CFRP no reforço ao corte de vigas de BA: investigação experimental e modelos analíticos (CFRP for the shear strengthening of RC beams: Experimental and analytical research)", Technical Report 04-DEC/E-08, Dep. Civil Eng., School of Eng., University of Minho, May 2004, 108 pp. (in Portuguese)

[13] R. A. Cook, G. T. Doerr, and R. E. Klingner, "Bond Stress Model for Design of Adhesive Anchors", ACI Structural Journal, Vol. 90(5), pp.514-524, September-October 1993.

[14] R. A. Cook, A. Ronal, K. Jacob, F. Werner, R. Konz, C. Robert, "Behaviour and Design of Single Adhesive Anchors under Tensile Load in Uncracked Concrete", ACI Structural Journal, Vol. 95(1), pp. 9-26, January/February 1998.

[15] R. A. Cook, and R., Konz, "Factors Influencing the Bond Strength of Adhesive Anchors," ACI Structural Journal, American Concrete Institute (ACI), Vol. 98(1), pp. 76-86, January-February 2001.

[16] CEB bulletin d'information $\mathrm{N}^{\circ} 233$ "Design of Fastenings in Concrete - Design Guide" - Parts 1 to 3, printed revised hardbound edition of Bulletin 226 part 1, Telford, London, 1997; p. 83.

[17] J. M. Sena-Cruz, J. A. O. Barros, A. F. M. Azevedo, R. Gettu, "Bond behavior of near-surface mounted CFRP laminate strips under monotonic and cyclic loading", Journal of Composites for Construction, ASCE, Vol. 10(4), pp. 295-303, 2006.

[18] J.G. Teng, L. De Lorenzis, B. Wong, R. Li, T.N. Wang, L. Lam, "Debonding failures of RC Beams Strengthened with Near Surface Mounted CFRP Strips", Journal of Composites for Construction, ACSE, Vol. 10(2), pp. 92-105, March-April 2006.

[19] CEB-FIP, Model Code 1990, Comite Euro-International du Beton, Bulletin d'Information nº 213/214, T. Telford, Ed., London, 1993. 